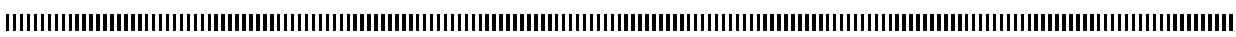

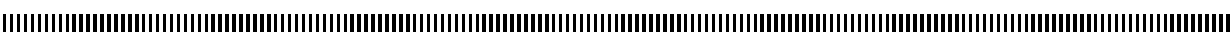

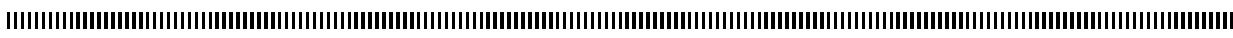

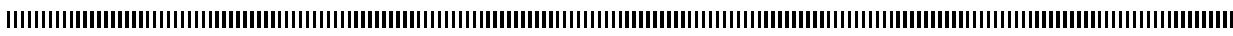

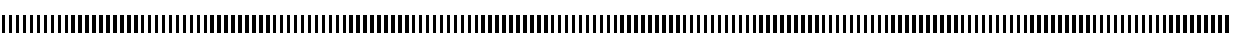
| |

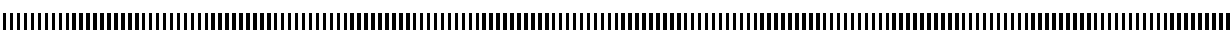
| |

\title{
Composite Asymptotic Expansions and Difference Equations
}

\author{
Augustin Fruchard* — Reinhard Schäfke** \\ * Laboratoire de Mathématiques, Informatique et Applications, EA 3993 \\ Faculté des Sciences et Techniques, Université de Haute Alsace 4, rue des Frères Lumière \\ 68093 Mulhouse cedex \\ France \\ Augustin.Fruchard@uha.fr \\ ** Institut de Recherche Mathématique Avancée, UMR 7501 \\ U.F.R. de Mathématiques et Informatique \\ Université Louis Pasteur et C.N.R.S. \\ 7, rue René Descartes \\ 67084 Strasbourg cedex \\ France \\ schaefke@math.u-strasbg.fr
}

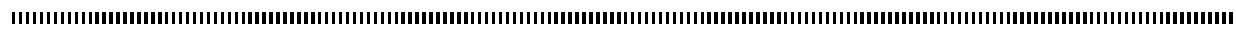

ABSTRACT. Difference equations in the complex domain of the form $y(x+\varepsilon)-y(x)=\varepsilon f(y(x)) / y(x)$ are considered. The step size $\varepsilon>0$ is a small parameter, and the equation has a singularity at $y=0$. Solutions near the singularity are described using composite asymptotic expansions. More precisely, it is shown that the derivative $v^{\prime}$ of the inverse function $v$ of a solution (the so-called Fatou coordinate) admits a Gevrey asymptotic expansion in powers of the square root of $\varepsilon$, denoted by $\eta$, involving functions of $y$ and of $Y=y / \eta$. This also yields Gevrey asymptotic expansions of the so-called EcalleVoronin invariants of the equation which are functions of $\varepsilon$. An application coming from the theory of complex iteration is presented.

RÉSUMÉ. On considère des équations aux différences dans le plan complexe de la forme $y(x+$ $\varepsilon)-y(x)=\varepsilon f(y(x)) / y(x)$. Le pas de discrétisation $\varepsilon>0$ est un petit paramètre, et l'équation a une singularité en $y=0$. On décrit les solutions près de la singularité en utilisant des développements asymptotiques combinés. Plus précisément, on montre que la dérivée $v^{\prime}$ de la fonction réciproque (appelée coordonnée de Fatou) $v$ d'une solution admet un développement asymptotique Gevrey en puissances de la racine carrée de $\varepsilon$, notée $\eta$, et faisant intervenir des fonctions de $y$ et de $Y=y / \eta$. On obtient également des développements asymptotiques Gevrey des invariants d'Écalle-Voronin de l'équation, qui sont des fonctions de $\varepsilon$. Une application venant de la théorie de l'itération complexe est présentée.

KEYWORDS : difference equation with small step size, composite asymptotic expansion, Gevrey asymptotic, Fatou coordinate, Ecalle-Voronin invariants.

MOTS-CLÉS : Équation aux différences à petit pas, développement asymptotique combiné, asymptotique Gevrey, coordonnée de Fatou, invariant d'Écalle-Voronin. 


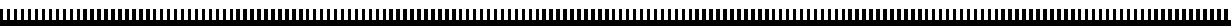

\section{Introduction.}

The main purpose of this article is to assemble two theories, which match each other particularly well, in order to obtain new results on solutions of a difference equation with singularity. The first theory, difference equations with small step size in the complex domain, is developed in [3]. It concerns equations of the form

$$
\Delta_{\varepsilon} y=f(x, y, \varepsilon)
$$

where $f: \Omega \subseteq \mathbb{C} \times \mathbb{C}^{N} \times\left[0, \varepsilon_{0}\right] \rightarrow \mathbb{C}^{N}$ is holomorphic in $x$ and $y$ and continuous in $\varepsilon$, $\varepsilon>0$ is a small parameter, and $\Delta_{\varepsilon}$ is the difference operator given by

$$
\Delta_{\varepsilon} y(x)=\frac{1}{\varepsilon}(y(x+\varepsilon)-y(x)) .
$$

The first main result of [3] is that, on horizontally convex domains, there exist solutions of (1.1) close to any solution of the limiting differential equation

$$
y^{\prime}=f(x, y, 0) .
$$

A domain of $\mathbb{C}$ is called horizontally convex if, for all its points $x, x^{\prime}$ with $\operatorname{Im} x=\operatorname{Im} x^{\prime}$, the segment $\left[x, x^{\prime}\right]$ is contained in it. More precisely, given $y_{0}: D \rightarrow \mathbb{C}^{N}$ a solution of (1.2) holomorphic on some $x$-domain $D$ such that $\left(x, y_{0}(x), \varepsilon\right) \in \Omega$ for all $x \in D$ and all $\varepsilon \in\left[0, \varepsilon_{0}\right]$, given an initial condition $\left(x_{0}, d(\varepsilon)\right), d:\left[0, \varepsilon_{0}\right] \rightarrow \mathbb{C}^{N}$ continuous with $d(0)=y_{0}\left(x_{0}\right)$, and given a horizontally convex domain $H$ compactly contained in $D$, it is shown in [3] that there exist $\left.\varepsilon_{1} \in\right] 0, \varepsilon_{0}$ ] and a family of solutions $\left.\left.y: D \times\right] 0, \varepsilon_{1}\right] \rightarrow \mathbb{C}^{N}$ of (1.1), continuous, holomorphic in $x$, such that $y\left(x_{0}, \varepsilon\right)=d(\varepsilon)$ and $y(x, \varepsilon)=y_{0}(x)+$ $\mathcal{O}(\varepsilon)$ on $H$.

A second result of [3] is that two solutions of (1.1) which coincide at some point of $D$ are exponentially close one to each other on the domain $D$. The last main result is that, provided $f$ is holomorphic in $\varepsilon$ in a complex neighborhood of 0 and $d(\varepsilon)$ has an asymptotic expansion $d(\varepsilon) \sim \sum_{n>0} d_{n} \varepsilon^{n}$ as $\varepsilon \rightarrow 0$, the above solutions $y$ have an asymptotic expansion $\sum_{n \geq 0} y_{n}(x) \varepsilon^{n}$ as $\varepsilon \rightarrow 0$ uniformly on $D$, where the coefficients $y_{n}$ are holomorphic functions on $D$ and can be determined recursively as solutions of certain initial value problems of the form

$$
y_{n}^{\prime}=\frac{\partial f}{\partial y}\left(x, y_{0}(x), 0\right) y_{n}+F_{n}\left(x, y_{0}(x), \ldots, y_{n-1}(x)\right), \quad y_{n}\left(x_{0}\right)=d_{n} .
$$

The second theory in the title of the present article, composite asymptotic expansions, is developed in [4]. It deals with asymptotic expansions of functions of two variables $x$ and $\eta$, using at the same time functions of $x$ and functions of the quotient $\frac{x}{\eta}$.

At the origin this theory was developed to study singularly perturbed differential equations of the form

$$
\varepsilon y^{\prime}=f(x) y+\varepsilon P(x, y, \varepsilon)
$$


near a turning point. Here a turning point is a zero of the function $f$. Without loss, we assume that this turning point is at the origin $x=0$. Let the integer $p \geq 2$ be such that the order of the zero of $f$ at $x=0$ is $p-1$, and let $\eta=\varepsilon^{1 / p}$. One of the main results of [4] is that there exists a solution $y$ of (1.5) defined for $\varepsilon$ in a sector

$$
S\left(-\delta, \delta, \varepsilon_{0}\right)=\left\{\varepsilon \in \mathbb{C} ;|\varepsilon|<\varepsilon_{0} \text { and }-\delta<\arg \varepsilon<\delta\right\}
$$

and for $x$ in a so-called quasi-sector $V(\alpha, \beta, r, \mu|\eta|), \mu<0$ (hence depending on $|\eta|=$ $\left.|\varepsilon|^{1 / p}\right)$, where

$$
V(\alpha, \beta, r, \rho)=\{x \in \mathbb{C} ;-\rho<|x|<r \text { and } \alpha<\arg x<\beta\}
$$

with $\rho<0$, and that this solution has a composite asymptotic expansion (CASE for short) in the following sense. There exist a disc $D(0, r)$, an infinite quasi-sector $V=V(\alpha-$ $\delta, \beta+\delta, \infty, \mu)$, and holomorphic functions $a_{n}: D(0, r) \rightarrow \mathbb{C}$ and $g_{n}: V \rightarrow \mathbb{C}, g_{n}$ having an asymptotic expansion without constant term at infinity, such that

$$
y(x, \varepsilon) \sim_{\frac{1}{p}} \sum_{n \geq 0}\left(a_{n}(x)+g_{n}\left(\frac{x}{\eta}\right)\right) \eta^{n} .
$$

The symbol $\sim$ means that a partial sum up to order $N$ gives an approximation of the solution to order $\eta^{N}$, which is uniform in the whole domain $\varepsilon \in S\left(-\delta, \delta, \varepsilon_{0}\right), x \in$ $V(\alpha, \beta, r, \mu|\eta|)$. Therefore, formula (1.6) provides an approximation of the solution at the same time near the turning point and away from it, i.e. at distances of order $\eta$ from the turning point as well as at distances of order 1 . The extra symbol $\frac{1}{p}$ means that we also have estimates of Gevrey type for the remainders; some details can be found below Theorem 2.4.

In the present article, we use this theory of CAsEs in order to describe solutions of a difference equation with singularity. For the sake of simplicity, we consider an autonomous difference equation, i.e. with a right hand side depending only of $y$. We assume that the equation has a singularity. Fixing this singularity at 0 , we assume that $y=0$ is a simple pole of the right hand side. In other words, we consider a difference equation of the form

$$
\Delta_{\varepsilon} y=\frac{1}{y} f(y)
$$

where $f: U \subseteq \mathbb{C} \rightarrow \mathbb{C}$ is holomorphic in a domain $U$ containing $0, f(0) \neq 0$, and our purpose is to study the behavior of solutions of (1.7) having small values. Observe that the general theory described above applies on domains where the values of a solution are bounded away from 0 , but this theory no longer applies near points where the solution takes small values.

A new feature of this type of equations is that the limiting equations are of two different natures: Near the origin, the inner reduced equation (2.6) (see Section 2 below) is 
a difference equation, whereas far from the origin the outer reduced equation (2.2) is a differential equation. A natural question is whether an approximation of solutions of (1.7) exists, using solutions of the outer reduced equation (2.2) for $x$ of order 1 and solutions of the inner reduced equation (2.6) for $x$ of order $\varepsilon$, and which would be uniform, i.e. also for all intermediate $x$ small with respect to 1 and large with respect to $\varepsilon$.

Our CAsEs seem to be well adapted for this situation. It turns out, however, that we do not obtain CASEs for solutions of (1.7) but, except for a logarithmic term, for their inverse functions, called Fatou coordinates. Given a solution $y$ of (1.7), let $v=v(z, \varepsilon)$ denote the inverse function of $y$ with respect to the variable $x$, i.e. $z=y(x, \varepsilon) \Leftrightarrow x=v(z, \varepsilon)$. Then $v$ is a solution of the Schröder equation

$$
v\left(z+\varepsilon \frac{f(z)}{z}\right)=v(z)+\varepsilon .
$$

An indication why things are much simpler in Fatou coordinates than for the solutions themselves is the following. In the case of an autonomous equation of the form (1.7) the existence of one solution $y$ implies the existence of a family of solutions: If $y$ is a solution of (1.7) and $\tau \in \mathbb{C}$ is fixed, then the shifted function $y_{\tau}: x \mapsto y(x+\tau)$ is also a solution (one could even choose an $\varepsilon$-periodic function for $\tau$ ). If $y$ has an asymptotic expansion and $\tau$ depends on $\varepsilon$, then this expansion changes considerably under such a shift. If $v=v(z, \varepsilon)$ is the inverse function of $y$, then the inverse of the shifted solution $y_{\tau}$ is simply $v_{\tau}: z \mapsto v(z, \varepsilon)-\tau$; this changes the asymptotics not essentially.

In order to obtain CAsEs for the Fatou coordinates, we first construct solutions of (1.7), denoted $y_{1}, \ldots, y_{4}$, on some domains $\Omega_{j}$ which will be described in the sequel. Then we prove that they are invertible; the inverse functions $v_{j}=y_{j}^{-1},_{j}=1, \ldots, 4$, are solutions of the Schröder equation (1.8) defined on some domains containing quasi-sectors which cover an annulus $\{z \in \mathbb{C} ;-\mu|\eta|<|z|<r\}$, with some $\mu<0<r$ with $\eta=\varepsilon^{1 / 2}$. It turns out that the functions $v_{j+1} \circ v_{j}^{-1}$ are of the form id $+p_{j}$, with $p_{j}$ periodic of period $\varepsilon$. As a consequence we obtain exponentially small estimates for the differences $v_{j+1}^{\prime}-v_{j}^{\prime}$ of the derivatives. Using a Ramis-Sibuya-type theorem we then obtain a CAsE for the derivative $v^{\prime}$ of the Fatou coordinate. By integration, this yields a CAsE for $v$, except for a logarithmic term. Using some inversion of CAsEs, it might be possible to deduce a CASE for the solution $y$ itself, but this CAsE would contain powers of $\log x$ of any order. To sum up, an approximation of the solution $y$ would be much more complicated than the approximation of its inverse.

We are particularly interested in the so-called Écalle-Voronin invariants [2, 7] of equation (1.7). These invariants are the Fourier coefficients of the periodic functions $p_{j}$. They play an important role in the theory of analytic equivalence of diffeomorphisms. The CAsEs for the $y_{j}$ yield also Gevrey asymptotic expansions for these invariants.

Application. In the last Section 7 we use our results in the special case of $f(y)=1+y$, i.e. the difference equation

$$
\Delta_{\varepsilon} y=1+\frac{1}{y} .
$$


This equation has also solutions on some infinite sectors, hence has also Écalle-Voronin invariants at infinity. The purpose of Section 7 is to compare these invariants with the Écalle-Voronin invariants at the origin. This study shows in particular that the first ÉcalleVoronin invariant at infinity, when extended to all arguments of $\varepsilon$, has an infinite number of zeroes, which are asymptotically in an arithmetical sequence close to the imaginary axis. Section 7 only sketches some ideas of proof; the complete proofs will appear in a future article.

Equation (7.1) appears in [1] in the form of the iteration of the diffeomorphism $F_{b}$ : $Z \mapsto Z+1+b / Z$ tangent to the identity at $Z=\infty$, i.e. with $b=\frac{1}{\varepsilon}$ and $Z=\frac{y}{\varepsilon}$ in our notation. The authors prove that the first Écalle-Voronin invariant, denoted by $\mathcal{C}_{r / s}(b)$, an entire function of $b$, has super-exponential growth as $|b|$ tends to infinity which implies that it has infinitely many zeros. Because of different normalizations, the link between their invariant and ours contains an additional term $\exp (2 \pi i b \log b)$. This explains the different growth of the two functions and this could also be used to prove the superexponential growth of $\mathcal{C}_{r / s}(b)$.

Beyond this last result, our motivation to study equation (2.1) was to illustrate our theory of CASEs in a context where the reduced outer and inner equations are of different kinds, here a differential outer equation and a difference inner equation. We believe that our CASES can be useful for other types of functional equations, e.g. partial differential equations or other functional equations where small (or large) parameters occur.

\section{Statements of the main results.}

We consider the difference equation with small step size (1.7) rewritten below for convenience

$$
\Delta_{\varepsilon} y=\frac{1}{y} f(y)
$$

where $f: U \subseteq \mathbb{C} \rightarrow \mathbb{C}$ is holomorphic in a domain $U$ containing $0, f(0)=\frac{\alpha^{2}}{2} \neq 0$, $\varepsilon>0$ is a small parameter, and $\Delta_{\varepsilon}$ is the difference operator given by (1.2). Equation (2.1) has two limiting equations. The first one is the so-called outer reduced equation

$$
y^{\prime}=\frac{1}{y} f(y)
$$

obtained when $\varepsilon$ tends to 0 . One easily checks that equation (2.2) has a unique solution $y_{0}$, defined in a neighborhood of 0 on the two-sheet Riemann surface $\Sigma$ of the square root, such that $y_{0}(x) \sim \alpha \sqrt{x}$ as $x \rightarrow 0$ on $\Sigma$. This solution is given implicitely by $x=a_{0}\left(y_{0}(x)\right)$, with

$$
a_{0}(y)=\int_{0}^{y} \frac{t d t}{f(t)}
$$


Our purpose is to study the behavior of solutions taking small values near $x=0$. A first idea is to perform the change of variables $x=\varepsilon X, y=\eta Y$ with $\eta=\sqrt{\varepsilon}$, i.e.

$$
Y(X)=\frac{1}{\eta} y(\varepsilon X) \text {. }
$$

This transforms (2.1) into the equation

$$
\Delta_{1} Y=\frac{1}{Y} f(\eta Y)
$$

whose limit, as $\eta \rightarrow 0$, is the second limiting equation, the inner reduced equation

$$
Y(X+1)=Y(X)+\frac{\alpha^{2}}{2 Y(X)} .
$$

Given $\delta>0$ small enough and $K>0$ large enough, consider the sector

$$
\Omega_{+}(K, \delta)=\{X \in \mathbb{C} ;|\arg (X-K)|<\pi-\delta\},
$$

and let $Q_{+}(K, \delta)$ denote the image of $\Omega_{+}(K, \delta)$ by the function $X \mapsto \alpha X^{1 / 2}$, see Figure 2.1 for a sketch. Here log is the principal determination of the logarithm on $\Omega_{+}(K, \delta)$, and $X^{a}=\exp (a \log X)$.
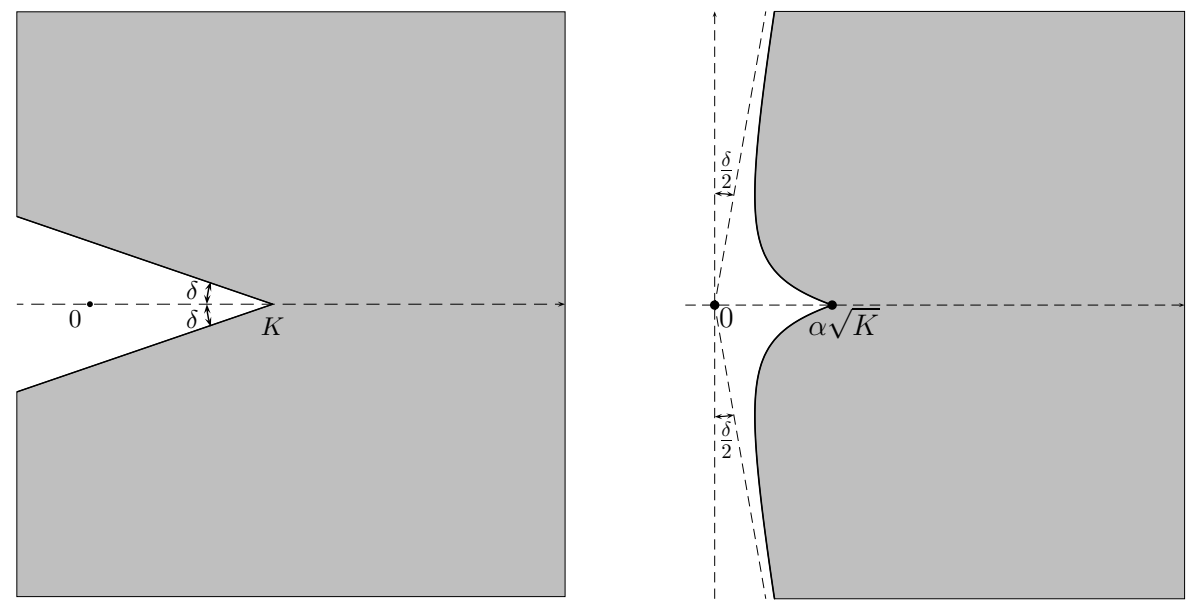

Figure 2.1. The sector $\Omega_{+}(K, \delta)$ and its image $Q_{+}(K, \delta)$ by $X \mapsto \alpha X^{1 / 2}$ in the case $\alpha>0$.

Concerning the inner reduced equation (2.6), we have the following result.

Proposition 2.1 . For all $\delta>0$ there exists $K>0$ such that (2.6) has a unique solution $Y_{+}$defined on $\Omega_{+}=\Omega_{+}(K, \delta)$ satisfying

$$
Y_{+}(X)=\alpha X^{1 / 2}+\frac{\alpha}{8} X^{-1 / 2} \log X+o\left(X^{-1 / 2}\right), \quad X \rightarrow \infty, X \in \Omega_{+} .
$$


If $K$ is large enough, then the function $Y_{+}$has an inverse function $V_{+}: Q_{+}(2 K, 2 \delta) \rightarrow$ $\Omega_{+}(K, \delta)$ which satisfies

$$
V_{+}(Z)=\left(\frac{Z}{\alpha}\right)^{2}-\frac{1}{2} \log \left(\frac{Z}{\alpha}\right)+o(1), Q_{+}(2 K, 2 \delta) \ni Z \rightarrow \infty
$$

and the functional equation

$$
V\left(Z+\frac{\alpha^{2}}{2 Z}\right)=V(Z)+1
$$

whenever $Z$ and $Z+\frac{\alpha^{2}}{2 Z}$ are in $Q_{+}(2 K, 2 \delta)$.

Remarks. 1. This kind of statement is very classical. For the sake of completeness, heowever, a detailed proof is given in Section 3.

2. More precisely one has

$$
Y_{+}(X)=\alpha X^{1 / 2}+\frac{\alpha}{8} X^{-1 / 2} \log X+\mathcal{O}\left(X^{-3 / 2}(\log X)^{2}\right), \Omega_{+} \ni X \rightarrow \infty
$$

and the derivative of $Y_{+}$satisfies

$$
Y_{+}^{\prime}(X)=\frac{\alpha}{2} X^{-1 / 2}+\mathcal{O}\left(X^{-3 / 2}(\log X)^{2}\right), \Omega_{+} \ni X \rightarrow \infty .
$$

3. The function $V_{+}$is a so-called Fatou coordinate of (2.6).

By symmetry of (2.6), it follows that $-Y_{+}$is the only solution $Y$ of (2.6) on $\Omega^{+}$ satisfying $Y(X)=-\alpha X^{1 / 2}-\frac{\alpha}{8} X^{-1 / 2} \log X+o\left(X^{-1 / 2}\right)$. Its inverse is the function $Z \mapsto V_{+}(-Z)$ defined on $-Q_{+}(2 K, 2 \delta)$; it also satisfies (2.9).

For $K$ large enough, one proves in a similar way that there exists a unique function $Y_{-}$defined on $\Omega_{-}=\{X \in \mathbb{C} ;|\arg (-X-K)|<\pi-\delta\}$ satisfying

$$
Y_{-}(X)=\alpha X^{1 / 2}+\frac{\alpha}{8} X^{-1 / 2} \log X+o\left(X^{-1 / 2}\right) \text { as } X \rightarrow \infty \text { in } \Omega_{-},
$$

and satisfying (2.6) for all $X \in \Omega_{-}$such that $X+1 \in \Omega_{-}$. Here $\log X$ is the analytic continuation of the principal branch onto $\Omega_{-}$in the mathematically positive direction, i.e. $\log X=\log (-X)+\pi i$. In the same way, we continue the roots analytically by $X^{ \pm 1 / 2}=$ $\pm i(-X)^{ \pm 1 / 2}$. For $K$ large enough, we have again an inverse $V_{-}$of $Y_{-}$defined on $Q_{-}(2 K, 2 \delta)=i Q_{+}(2 K, 2 \delta)$ that satisfies (2.9) and $V_{-}(Z)=\left(\frac{Z}{\alpha}\right)^{2}-\frac{1}{2} \log \left(\frac{Z}{\alpha}\right)+o(1)$. In order to prove these statements, the proof in Section 2 has to be modified essentially only at one point: The operator $\mathbf{T}$ in (3.3) has to be defined using summation over all $X-n, n$ positive integer.

As another solution of (2.6), we consider $-Y_{-}$. It is also defined on $\Omega_{-}$and satisfies $-Y_{-}(X)=-\alpha X^{1 / 2}-\frac{\alpha}{8} X^{-1 / 2} \log X+o\left(X^{-1 / 2}\right)$. Its inverse is the function $Z \mapsto V_{-}(-Z)$ defined on $-Q_{-}(2 K, 2 \delta)$; it also satisfies (2.9). In this manner, we have obtained four solutions of (2.6) and four solutions of (2.9) of particular interest. 
If $K$ is large enough, the function $\Phi_{+}=V_{-} \circ Y_{+}-$id is defined (at least) on the sector

$$
\mathcal{I}_{+}=\left\{X \in \mathbb{C} ;\left|\arg (X-i K)-\frac{\pi}{2}\right|<\frac{\pi}{2}-3 \delta\right\} .
$$

Using (2.9), it is easily shown that $\Phi_{+}$is 1-periodic. The choice of the branches of the logarithms in Proposition 2.1 and the estimate (2.8) ensure that $\Phi_{+}(X) \rightarrow 0$ as $\mathcal{I}_{+} \ni$ $X \rightarrow \infty$. Therefore the Fourier series of $\Phi_{+}$must have the following form

$$
\Phi_{+}(X)=\sum_{n=1}^{\infty} C_{n}^{+} e^{2 \pi i n X} \text { for } X \in \mathcal{I}_{+},
$$

where $C_{n}^{+} \in \mathbb{C}$ are constants.

Similarly, we treat the composition of the inverse of $Y^{+}$with $-Y^{-}$. The function $\Phi_{-}=V_{+} \circ\left(-Y_{-}\right)-$id is defined (at least) on the sector

$$
\mathcal{I}_{-}=\left\{X \in \mathbb{C} ;\left|\arg (X+i K)+\frac{\pi}{2}\right|<\frac{\pi}{2}-3 \delta\right\} .
$$

It is also 1-periodic, but only bounded as $\mathcal{I}_{-} \ni X \rightarrow \infty$ because of the choice of the branches of the logarithms. Its Fourier series is thus

$$
\Phi_{-}(X)=\sum_{n=0}^{\infty} C_{n}^{-} e^{-2 \pi i n X} \text { for } X \in \mathcal{I}_{-} .
$$

Here $C_{0}^{-}=\frac{\pi i}{2}$; the other constants $C_{n}^{-}, n \geq 1$ are closely related to $C_{n}^{+}$, but the relation is not interesting in our work. The other analogous compositions of the inverse of $-Y^{-}$ with $-Y^{+}$, respectively that of the inverse of $Y^{+}$with $-Y^{-}$, are identical to $\Phi^{ \pm}+$id with the above functions $\Phi^{ \pm}$and yield no new constants.

The constants $C_{n}^{ \pm}$are the so-called Écalle-Voronin invariants [2, 7] of equation (2.6).

Let us return to our original equation (2.1). We fix $K, r, \delta>0$. Let $z(\varepsilon) \in[-r, i r]$ be such that $\arg (z(\varepsilon)-K \varepsilon)=\pi-\delta$ and let $\Omega_{1}=\Omega(K, r, \delta, \varepsilon)$ denote the interior of the (non convex) hexagon with vertices $K \varepsilon, z(\varepsilon), i r, r,-i r, \bar{z}(\varepsilon)$ in this order; see Figure 2.2. Let $\Omega_{2}=\Omega_{4}=-\Omega_{1}=\left\{x \in \mathbb{C} ;-x \in \Omega_{1}\right\}$ and $\Omega_{3}=\Omega_{1}$.

We will also use the image of $\Omega_{1}$ by $x \mapsto \alpha x^{1 / 2}$, denoted by $Q_{1}$, and the domains $Q_{j}=i^{j-1} Q_{1}, j=2,3,4$, obtained by rotations.

Our first main result is as follows; let us recall that $\eta=\varepsilon^{1 / 2}$.

Theorem 2.2. With the above notation, for all $\delta>0$ there exist $K, R, \varepsilon_{0}, r>0$ with $K \varepsilon_{0}<r$ and four solutions $y_{1}, y_{2}, y_{3}, y_{4}$ of (2.1), defined for $\left.\left.\varepsilon \in\right] 0, \varepsilon_{0}\right]$ and $x \in \Omega_{j}$, such that $y_{1}(K \varepsilon, \varepsilon)=-y_{3}(K \varepsilon, \varepsilon)=\eta Y_{+}(K), y_{2}(-K \varepsilon, \varepsilon)=-y_{4}(-K \varepsilon, \varepsilon)=\eta Y_{-}(-K)$, and

$\left.\forall \varepsilon \in] 0, \varepsilon_{0}\right] \forall x \in \Omega_{1}, \quad\left|y_{1}(x, \varepsilon)-\eta Y_{+}\left(\frac{x}{\varepsilon}\right)\right| \leq R|x|$ and $\left|y_{3}(x, \varepsilon)+\eta Y_{+}\left(\frac{x}{\varepsilon}\right)\right| \leq R|x|$, $\left.\forall \varepsilon \in] 0, \varepsilon_{0}\right] \forall x \in-\Omega_{1},\left|y_{2}(x, \varepsilon)-\eta Y_{-}\left(\frac{x}{\varepsilon}\right)\right| \leq R|x|$ and $\left|y_{4}(x, \varepsilon)+\eta Y_{-}\left(\frac{x}{\varepsilon}\right)\right| \leq R|x|$. 

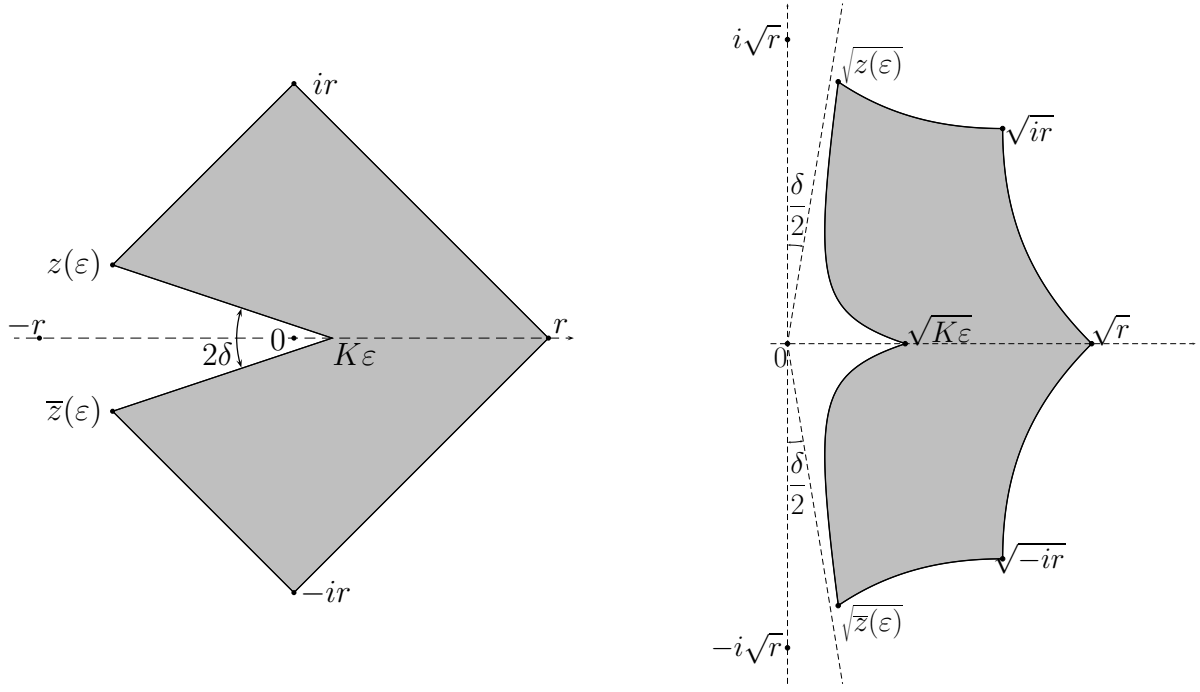

Figure 2.2. Left: The domain $\Omega_{1}$ of existence of the solutions $y_{1}, y_{3}$ of (2.1). Right: The image $Q_{1}$ of $\Omega_{1}$ by $x \mapsto x^{1 / 2}$ ( $\alpha=1$ here).

The proof is in Section 5. Since the domains are no longer infinite, we cannot have uniqueness of the solutions anymore, but they are unique up to exponentially small terms.

We then prove the existence of the inverse functions $v_{j}=y_{j}^{-1}$ analogous to the above Fatou coordinates. Precisely, let $\widetilde{\Omega}_{1}=\Omega\left(2 K, \frac{r}{2}, 2 \delta, \varepsilon\right)$ be defined as $\Omega_{1}$, with the constants $2 K, \frac{r}{2}$, and $2 \delta$ instead of $K, r, \delta$. We assume $\varepsilon_{0} \leq \frac{r}{4 K}$. Let $\widetilde{Q}_{1}$ denote the image of $\widetilde{\Omega}_{1}$ by the function $X \mapsto \alpha X^{1 / 2}$. As before, we also use $\widetilde{\Omega}_{j}=(-1)^{j-1} \widetilde{\Omega}_{1}$ and $\widetilde{Q}_{j}=i^{j-1} \widetilde{Q}_{1}, j=2,3,4$. On $\widetilde{Q}_{1}$, we use the principal value of $\log \left(\frac{z}{\alpha \eta}\right)$; on the other $\widetilde{Q}_{j}$, we use $\log \left(\frac{z}{\alpha \eta}\right)=\log \left(\frac{z}{\alpha \eta} i^{1-j}\right)+\frac{j-1}{2} \pi i$. Thus the branches of the logarithms are the same on the intersection $\widetilde{Q}_{j} \cap \widetilde{Q}_{j+1}$ if $j=1,2,3$, but not on $\widetilde{Q}_{4} \cap \widetilde{Q}_{1}$.

Proposition 2.3 . With the above notation, if $j \in\{1,2,3,4\}, r>0$ is small enough and $K>0$ is large enough then, for all $z \in \widetilde{Q}_{j}$, the equation $y_{j}(x, \varepsilon)=z$ has a unique solution $x \in \Omega_{j}$, denoted by $x=v_{j}(z, \varepsilon)$. This gives a holomorphic function $v_{j}$ defined for $\left.\left.z \in \widetilde{Q}_{j}, \varepsilon \in\right] 0, \varepsilon_{0}\right]$, the values of which are in $\Omega_{j}$. It is a solution of

$$
v\left(z+\varepsilon \frac{f(z)}{z}\right)=v(z)+\varepsilon .
$$

Moreover, we have

$$
\begin{array}{ll}
v_{1}\left(\eta Y_{+}(K), \eta^{2}\right)=K \eta^{2}, & v_{2}\left(\eta Y_{-}(-K), \eta^{2}\right)=-K \eta^{2} \\
v_{3}\left(-\eta Y_{+}(K), \eta^{2}\right)=K \eta^{2}, & \text { and } \\
v_{4}\left(-\eta Y_{-}(-K), \eta^{2}\right)=-K \eta^{2}
\end{array}
$$


where $K$ is the constant of Theorem 2.2, and there exist $R, \eta_{0}>0$ such that for all $\left.\left.z \in \widetilde{Q}_{j}, \eta \in\right] 0, \eta_{0}\right]$

$$
\begin{aligned}
& \left|v_{1}\left(z, \eta^{2}\right)-\eta^{2} V_{+}\left(\frac{z}{\eta}\right)\right| \leq R|z|^{3}, \quad\left|v_{3}\left(z, \eta^{2}\right)-\eta^{2} V_{+}\left(-\frac{z}{\eta}\right)\right| \leq R|z|^{3}, \\
& \left|v_{2}\left(z, \eta^{2}\right)-\eta^{2} V_{-}\left(\frac{z}{\eta}\right)\right| \leq R|z|^{3} \text {, and }\left|v_{4}\left(z, \eta^{2}\right)-\eta^{2} V_{-}\left(-\frac{z}{\eta}\right)\right| \leq R|z|^{3} \text {. }
\end{aligned}
$$

The proof is analogous to that of Proposition 2.1; it will be omitted here.

For fixed $z \neq 0$ in the appropriate domains, the limits $v_{j}(z, 0)=\lim _{\varepsilon \rightarrow 0} v_{j}(z, \varepsilon)$ are solutions of $v^{\prime}(z) \frac{f(z)}{z}=1$; this is obtained easily from (2.11) in the limit $\varepsilon \rightarrow 0$. The approximation conditions of the above proposition imply that $v_{j}(z, 0)=a_{0}(z)$, where $a_{0}$ is given in (2.3).

The approximation conditions of the above proposition also imply that, for fixed $Z$ sufficiently large such that $\eta Z$ is in the appropriate domain, $\lim _{\eta \rightarrow 0} \eta^{-2} v_{j}\left(\eta Z, \eta^{2}\right)$ is one of the four functions $V_{ \pm}( \pm Z)$.

Thus we have outer approximations (for $z$ fixed) and inner approximations (for $Z$ fixed) for $v_{j}$. The most important result of our article refines these statements, not only to the existence of full outer and inner expansions, but to full uniform expansions in the whole domains $\widetilde{Q}_{j}$. This is achieved using so-called composite asymptotic expansions (CASEs). We refer to [4] for a detailed discussion of this notion and its properties. Nevertheless, we will give explanations below the theorem. In the present article, we adopt the notation $b_{n}^{j}$ of [4] in case of two indices, with one index in superscript; we hope this will not bring confusion to the reader with the usual powers $\eta^{n}, i^{j-1}$, etc.

Theorem 2.4. The Fatou coordinates $v_{j}$ of (2.1) have composite asymptotic expansions (CASEs) of Gevrey order $\frac{1}{2}$ :

$$
v_{j}\left(z, \eta^{2}\right) \sim_{\frac{1}{2}} a_{0}(z)+S(\eta) \log \left(\frac{z}{\alpha \eta}\right)+T_{j}(\eta)+\sum_{n \geq 2}\left(a_{n}(z)+b_{n}^{j}\left(\frac{z}{\eta}\right)\right) \eta^{n}
$$

as $0<\eta \rightarrow 0$ uniformly for $z \in \widetilde{Q}_{j}$, where $a_{n}$ are analytic on $|z|<r / 2, a_{n}(0)=0$ and $b_{n}^{j}$ are holomorphic on $i^{j-1} Q_{+}(2 K, 2 \delta)$, cf. above Proposition 2.1. The latter have consistent asymptotic expansions of Gevrey order $\frac{1}{2}$

$$
b_{n}^{j}(Z) \sim_{\frac{1}{2}} \sum_{m \geq 1} B_{n m} Z^{-m} \text { as } Z \rightarrow \infty .
$$

Furthermore, the functions $S, T_{j}$ admit asymptotic expansions of Gevrey order $\frac{1}{2}$ :

$$
S(\eta) \sim_{\frac{1}{2}} \sum_{n \geq 1} S_{n} \eta^{2 n}, \quad T_{j}(\eta) \sim_{\frac{1}{2}} \sum_{n \geq 2} T_{j n} \eta^{n},
$$

the function $a_{0}$ is given in (2.3) and the functions $b_{0}^{j}, b_{1}^{j}$ and $a_{n}, n$ odd, are identically zero. Moreover, we have $S_{1}=-\frac{1}{2}, T_{12}=T_{22}=0, T_{32}=T_{42}=\frac{1}{2} \pi i$. 
By definition, (2.12) means that there exist $A, B, \eta_{0}>0$ such that, for all $\left.\left.\eta \in\right] 0, \eta_{0}\right]$, all $z \in \widetilde{Q}_{j}$, and all $N \in \mathbb{N}, N \geq 2$, one has

$$
\begin{array}{r}
\left|v_{j}\left(z, \eta^{2}\right)-a_{0}(z)-S(\eta) \log \left(\frac{z}{\alpha \eta}\right)-T_{j}(\eta)-\sum_{n=2}^{N-1}\left(a_{n}(z)+b_{n}^{j}\left(\frac{z}{\eta}\right)\right) \eta^{n}\right| \\
A B^{N} \Gamma\left(1+\frac{N}{2}\right) \eta^{N} .
\end{array}
$$

The statement on the $b_{n}^{j}$ means that there exist $A, B>0$ such that, for all integers $n \geq$ 2, $M \geq 1$ and all $Z \in i^{j-1} Q_{+}(2 K, 2 \delta)$, one has

$$
|Z|^{M}\left|b_{n}^{j}(Z)-\sum_{m=1}^{M-1} B_{n m} X^{-m}\right| \leq A B^{n+M} \Gamma\left(\frac{M+n}{2}+1\right) .
$$

Observe that the $a_{n}, B_{n m}$ and $S$ are independent of $j$, whereas the $b_{n}^{j}$ and $T_{j}$ are not.

An important consequence of (2.12) (see [4], Proposition 3.7) is the existence of socalled outer and inner expansions of $v_{j}$ of Gevrey order $\frac{1}{2}$. More precisely, for every $\left.r_{1} \in\right] 0, \frac{r}{2}[$

$$
v_{j}\left(z, \eta^{2}\right) \sim_{\frac{1}{2}} a_{0}(z)+S(\eta) \log \left(\frac{z}{\alpha}\right)-S(\eta) \log \eta+T_{j}(\eta)+\sum_{n \geq 2} d_{n}(z) \eta^{n}
$$

as $\eta>0, \eta \rightarrow 0$ uniformly for $z \in \widetilde{Q}_{j}$ with $|z|>r_{1}$, where $d_{n}(z)=a_{n}(z)+$ $\sum_{m=1}^{n-2} B_{n-m, m} z^{-m}$, and for every $K_{1}>2 K$

$$
v_{j}\left(\eta Z, \eta^{2}\right) \sim_{\frac{1}{2}} S(\eta) \log \left(\frac{Z}{\alpha}\right)+T_{j}(\eta)+\sum_{n \geq 2} h_{n}^{j}(Z) \eta^{n}
$$

as $\eta>0, \eta \rightarrow 0$ uniformly for $\eta Z \in \widetilde{Q}_{j}$ with $2 K<|Z|<K_{1}$, where $h_{n}^{j}(Z)=$ $b_{n}^{j}(Z)+\sum_{m=1}^{n} A_{n-m, m} Z^{m}$ if $a_{j}(z)=\sum_{k>0} A_{j k} z^{k}$. Here we use that $a_{0}(z)=\mathcal{O}\left(z^{2}\right)$ and thus we also have $A_{00}=A_{01}=0$.

The proof of Theorem 2.4 is given in Section 6. In fact we first prove, using the main result of [4], that the derivatives $v_{j}^{\prime}(z, \varepsilon)$ of the Fatou coordinates have CASEs of Gevrey order $\frac{1}{2}$; here no logarithm appears. Because of the initial conditions of Proposition 2.3, we conclude for $v_{j}$ by integration; in the case $j=1$ for example, we have (with $\varepsilon=\eta^{2}$ )

$$
v_{1}(z, \varepsilon)=K \varepsilon+\int_{\eta Y_{+}(K)}^{z} v_{1}^{\prime}(\zeta, \varepsilon) d \zeta .
$$

The integration of a CASE is again treated in [4]; the logarithms appear because each term analogous to $b_{n}^{j}(Z)$ in this CASE contains a multiple of $1 / Z$ in its expansion. 
Remark. The right hand side of (2.12) is a composite formal series solution of (2.11). It can be shown that this determines the formal expression except for $T_{j}$; this will be done on an example in Section 7. The (Gevrey) asymptotic expansions of $T_{j}(\eta)$ are determined by the initial conditions of the $v_{j}$ (see below (2.11)) and the corresponding initial conditions (on a formal level) for the right hand sides of (2.12). Then the $T_{j}$ can be chosen by the Borel-Ritt-Gevrey theorem as any functions having these asymptotic expansions of Gevrey order $\frac{1}{2}$.

The additive constants $T_{j}(\eta)$ in the expansions depend upon the initial conditions of the $v_{j}$; especially they depend upon the choice of $K^{1}$. To avoid problems in the sequel, we want to normalize our solutions of (2.11) such that their Gevrey asymptotic expansions are uniquely determined by the equations and normalize the solutions of (2.1) accordingly - thus the functions are determined by the equation up to exponentially small terms. More precisely, we put for $j=1, \ldots, 4$

$$
v_{j}^{*}\left(z, \eta^{2}\right)=v_{j}\left(z, \eta^{2}\right)-T_{j}(\eta), y_{j}^{*}\left(x, \eta^{2}\right)=y_{j}\left(x+T_{j}(\eta), \eta^{2}\right) .
$$

Observe that $v_{j}^{*}$ is inverse to $y_{j}^{*}$ and that the domains of $v_{j}$ and $v_{j}^{*}$ are the same, whereas the domain of $y_{j}^{*}$ is obtained by shifting that of $y_{j}$. Here it is important that $T_{j}(\eta)=$ $\mathcal{O}\left(\eta^{2}\right)$ and thus the domain of $y_{j}^{*}$ is essentially of the same type as $\Omega_{j}$. In the sequel, we can assume without loss in generality that $y_{j}^{*}$ are defined on $\Omega_{j}$ and $v_{j}^{*}$ are defined on $\widetilde{Q}_{j}$ as defined above, provided $K$ is sufficiently large and $r>0$ is sufficently small.

It is easy to check that the functions $p_{j}=v_{j+1}^{*} \circ y_{j}^{*}-\mathbf{i d}, j=1, \ldots 4,{ }^{2}$ are $\varepsilon$ periodic in $x$. A priori these functions are defined on the sets $\left(y_{j}^{*}\right)^{-1}\left(\widetilde{Q}_{j+1}\right)=\{x \in$ $\left.\Omega_{j} ; y_{j}^{*}(x) \in \widetilde{Q}_{j+1}\right\}$. Their periodicity allows to continue them analytically to some strip $\left\{x \in \mathbb{C} ; \widetilde{K} \varepsilon<(-1)^{j-1} \operatorname{Im} x<\widetilde{r}\right\}$ with some $\widetilde{K}, \widetilde{r}>0$. The Fourier coefficients $c_{j n}$ of these functions, determined by

$$
p_{j}(x, \varepsilon)=\sum_{n \in \mathbb{Z}} c_{j n}(\varepsilon) e^{2 \pi i n x / \varepsilon}
$$

are called Écalle-Voronin invariants of (2.1). It turns out that $c_{j n}$ is exponentially small if $(-1)^{j-1} n$ is negative. For the other Écalle-Voronin invariants we have

Corollary 2.5 . If $(-1)^{j-1} k>0$, then the function $c_{j k}$ admits an asymptotic expansion $\sum_{n \geq 2} a_{j k n} \eta^{n}$ in powers of $\eta=\varepsilon^{1 / 2}$, where $a_{j k 2}$ is closely related to the first ÉcalleVoronin invariants of (2.6) defined in (2.10). More precisely, these are asymptotic expan-

1. They also depend upon the choice of the branch of $\log \left(\frac{z}{\alpha \eta}\right)$.

2. Here and in the sequel, the index $j+1$ is taken modulo 4 , i.e. $v_{5}^{*}=v_{1}^{*}$ etc. 
sions of Gevrey order $\frac{1}{2}$ in $\eta$ with closely related estimates, i.e. there exist $A, B>0$ such that, for all positive integers $N, k$,

$$
\left|c_{j k}(\varepsilon)-\sum_{n=2}^{N-1} a_{j k n} \eta^{n}\right| \leq A B^{N+k} \Gamma\left(1+\frac{N}{2}\right) \eta^{N} .
$$

If the branches of the logarithms are chosen as above Theorem 2.4 for $\widetilde{Q}_{j}$, then we have $a_{1 k 2}=C_{k}^{+}, a_{2,-k, 2}=C_{k}^{-}, a_{3 k 2}=e^{-k \pi^{2}} C_{k}^{+}$and $a_{4,-k, 2}=e^{k \pi^{2}} C_{k}^{-}$for positive integer $k$.

Idea of the proof. We indicate it only for $j=1$. We have

$$
c_{1 k}(\varepsilon)=\frac{1}{\varepsilon} \int_{x}^{x+\varepsilon} e^{-2 \pi i k \xi / \varepsilon}\left(v_{2}^{*} \circ y_{1}^{*}(\xi, \varepsilon)-\xi\right) d \xi .
$$

If $k<0$, then we can choose any $x$ in the strip with positive imaginary part (independent of $\varepsilon$ ) and we obtain that $c_{1 k}$ is exponentially small. If $k>0$, then the change of unknown $\xi=\varepsilon T, x=\varepsilon X$, with some fixed $X$ such that $\varepsilon[X, X+1]$ is in the domain of $v_{2} \circ y_{1}(., \varepsilon)$, yields

$$
c_{1 k}(\varepsilon)=\int_{X}^{X+1} e^{-2 \pi i k T}\left(v_{2}^{*} \circ y_{1}^{*}(\varepsilon T, \varepsilon)-\varepsilon T\right) d T .
$$

Now we use (2.16) and the inner expansions (2.15) for $v_{1}$ and $v_{2}$. Since the operations of composition and inversion are compatible with Gevrey asymptotic expansions, this yields a uniform asymptotic expansion of Gevrey order $\frac{1}{2}$ for $v_{2}^{*} \circ y_{1}^{*}(\varepsilon T, \varepsilon)-\varepsilon T$. The result follows easily integrating the expansion term by term.

Remark. Observe that the functions $\widetilde{p}_{j}=v_{j+1} \circ y_{j}-\mathbf{i d}$ defined using the non-normalized $v_{j}, y_{j}$ satisfy $\widetilde{p}_{j}(x, \varepsilon)=p_{j}\left(x-T_{j}(\eta), \varepsilon\right)+T_{j+1}(\eta)-T_{j}(\eta)$ and hence their Fourier coefficients $\widetilde{c}_{j n}(\varepsilon)$ are related to the above $c_{j n}(\varepsilon)$ by

$$
\begin{aligned}
\widetilde{c}_{j 0}(\varepsilon) & =c_{j 0}(\varepsilon)+T_{j+1}(\eta)-T_{j}(\eta), \\
\widetilde{c}_{j n}(\varepsilon) & =\exp \left(-2 \pi i n T_{j}(\eta) / \varepsilon\right) c_{j n}(\varepsilon) \text { if } n \neq 0 .
\end{aligned}
$$

\section{The reduced inner equation: Proof of Proposition 2.1.}

The change of unknown $Y(X)^{2}=\alpha^{2} X+\frac{\alpha^{2}}{4} \log X+U(X)$ in (2.6) yields the equation

$$
U(X+1)=U(X)+\frac{\alpha^{2}}{4} h(X, U(X))
$$

with

$$
h(X, U)=\left(X+\frac{1}{4} \log X+\alpha^{-2} U\right)^{-1}-\log \left(1+\frac{1}{X}\right) .
$$


Observe that, if $U$ is a solution of (3.1) satisfying $U(X)=o(1)$ as $\Omega_{+}(K, \delta) \ni X \rightarrow \infty$, then $h(X, U(X)) \sim-\frac{\log X}{4 X^{2}}$ as $\Omega_{+}(K, \delta) \ni X \rightarrow \infty$. By (3.1), $U$ is of the same order as the antiderivative of $h$ tending to 0 as $X \rightarrow \infty$, i.e. $U$ is of order $\frac{\log X}{X}$.

This leads us to introduce the following space. Let $\mathcal{E}$ denote the Banach vector space of functions $U$ holomorphic on $\Omega_{+}$such that $\frac{X U(X)}{\log X}$ is bounded, endowed with the norm

$$
\|U\|=\sup _{X \in \Omega_{+}}\left|\frac{X U(X)}{\log X}\right| .
$$

Given $L>0$ large enough, let $\mathcal{B}^{\prime}(0, L)$ denote the closed ball of $\mathcal{E}$ of center 0 and radius $L$, i.e. $\mathcal{B}^{\prime}(0, L)=\{U \in \mathcal{E} ;\|U\| \leq L\}$.

Using that $X+n \in \Omega_{+}$for all $X \in \Omega_{+}$and all $n \in \mathbb{N}$, we now rewrite (3.1) in a fixed point form $U=\mathbf{T} U$, with

$$
\mathbf{T} U(X)=-\frac{\alpha^{2}}{4} \sum_{n \geq 0} h(X+n, U(X+n))
$$

where $h$ is defined in (3.2). This latter sum converges for all $X \in \Omega_{+}$and all $U \in \mathcal{E}$ since $h(X, U(X))=\mathcal{O}\left(X^{-2} \log X\right)$.

Lemma 3.1 . For all $\delta>0$ and all $L \geq \frac{|\alpha|^{2}}{2 \sin ^{2}(\delta / 2)}$, there exists $K>0$ such that $\mathbf{T}: \mathcal{B}^{\prime}(0, L) \rightarrow \mathcal{B}^{\prime}(0, L)$ is a contraction.

Proof. As already seen, we have, for any fixed $L>0$ and any $U \in \mathcal{B}^{\prime}(0, L)$,

$$
h(X, U(X)) \sim-\frac{\log X}{4 X^{2}} \text { as } \Omega_{+} \ni X \rightarrow \infty,
$$

hence, for $K$ large enough, we have

$$
\forall U \in \mathcal{B}^{\prime}(0, L) \forall X \in \Omega_{+}, \quad|h(X, U(X))| \leq\left|X^{-2} \log X\right| .
$$

Now we use, for any $X \in \Omega_{+}$, that the quotient $\frac{X+n}{|X|+n}$ can be written as a convex combination of $\frac{X}{|X|}$ and 1 , namely

$$
\frac{X+n}{|X|+n}=\frac{|X|}{|X|+n} \cdot \frac{X}{|X|}+\frac{n}{|X|+n} \cdot 1
$$

hence has at least distance $\mu=\sin \frac{\delta}{2}$ from the origin. As a consequence, we have

$$
\forall X \in \Omega_{+} \forall n \in \mathbb{N}, \quad \mu(|X|+n) \leq|X+n| \leq|X|+n .
$$

If $K \sin \delta \geq 1$, we can also estimate, for all $X \in \Omega_{+}$and all $n \in \mathbb{N}$,

$$
|\log (X+n)| \leq \pi+\ln (|X|+n) .
$$


With (3.3), (3.4) and (3.5), this yields

$$
|\mathbf{T} U(X)| \leq \frac{|\alpha|^{2}}{4 \mu^{2}} \sum_{n \geq 0} \frac{\pi+\ln (|X|+n)}{(|X|+n)^{2}} .
$$

By a comparison of the sum and an integral, we estimate the sum of the right hand side of (3.6) by

$$
\frac{\ln |X|+\pi}{|X|^{2}}+\int_{|X|}^{+\infty} \frac{\pi+\ln t}{t^{2}} d t \leq \frac{2 \ln |X|}{|X|},
$$

if $K$ is large enough. Thanks to the condition on $L$ in the statement, we then obtain $|\mathbf{T} U(X)| \leq L \frac{\ln |X|}{|X|}$ for all $X \in \Omega_{+}$, i.e. $\|\mathbf{T} U\| \leq L$. Therefore $\mathbf{T}\left(\mathcal{B}^{\prime}(0, L)\right) \subseteq \mathcal{B}^{\prime}(0, L)$.

Now let $U, W \in \mathcal{B}^{\prime}(0, L) \subset \mathcal{E}$. Using that $\left|X+\frac{1}{4} \log X+\alpha^{-2} U(X)\right| \geq \frac{1}{2}|X|$ if $U \in \mathcal{B}^{\prime}(0, L), X \in \Omega_{+}$, and if $K$ is large enough, we estimate similarly

$$
\begin{aligned}
\mid h(X, U(X))-h & (X, W(X)) \mid \\
& =\left|\frac{\alpha^{-2}(W(X)-U(X))}{\left(X+\frac{1}{4} \log X+\alpha^{-2} U(X)\right)\left(X+\frac{1}{4} \log X+\alpha^{-2} W(X)\right)}\right| \\
& \leq 4 \alpha^{-2}|X|^{-2}|U(X)-W(X)| \\
& \leq 4 \alpha^{-2}|X|^{-3}|\log X|\|U-W\|
\end{aligned}
$$

hence

$$
|\mathbf{T} U(X)-\mathbf{T} W(X)| \leq \mu^{-3}\|U-W\| \sum_{n \geq 0}(|X|+n)^{-3}(\pi+\ln (|X|+n)) .
$$

By a comparison of the sum and an integral, we estimate the sum of the right hand side of (3.7) by

$$
|X|^{-3}(\pi+\ln |X|)+\int_{|X|}^{+\infty} t^{-3}(\pi+\ln t) d t \leq 2|X|^{-2} \ln |X| \leq 2|X|^{-2}|\log X|,
$$

if $K$ is large enough, hence $|\mathbf{T} U(X)-\mathbf{T} W(X)| \leq 2|X|^{-1} \mu^{-3}\|U-W\||X|^{-1}|\log X|$. Choosing $K$ such that $2|X|^{-1} \mu^{-3} \leq \frac{1}{2}$ for all $X \in \Omega_{+}$, we then obtain $\|\mathbf{T} U-\mathbf{T} W\| \leq$ $\frac{1}{2}\|U-W\|$, showing that $\mathbf{T}$ is a contraction in $\mathcal{B}^{\prime}(0, L)$.

Let us now return to the proof of Proposition 2.1. By lemma 3.1, there exists a (unique) solution $U_{+}$of (3.1) in the ball $\mathcal{B}^{\prime}(0, L)$ of $\mathcal{E}$. Then the function $Y_{+}$given by $Y_{+}(X)=$ $\left(\alpha^{2} X+\frac{\alpha^{2}}{4} \log X+U_{+}(X)\right)^{1 / 2}$ is a solution of (2.6) that satisfies

$$
Y_{+}(X)=\left(\alpha^{2} X+\frac{\alpha^{2}}{4} \log X+\mathcal{O}\left(\frac{\log X}{X}\right)\right)^{1 / 2}
$$




$$
=\alpha X^{1 / 2}\left(1+\frac{\log X}{8 X}+\mathcal{O}\left(\left(\frac{\log X}{X}\right)^{2}\right)+\mathcal{O}\left(\frac{\log X}{X^{2}}\right)\right) .
$$

If $Y_{1}$ is another solution of (2.6) satisfying $Y_{1}(X)=\alpha X^{1 / 2}+\frac{\alpha}{8} X^{-1 / 2} \log X+o\left(X^{-1 / 2}\right)$ as $\Omega_{+} \ni X \rightarrow \infty$, then the function $U_{1}$ given by $Y_{1}^{2}(X)=\alpha^{2} X+\frac{\alpha^{2}}{4} \log X+U_{1}(X)$ is a solution of (3.1) that satisfies $U_{1}(X)=o(1)$. It follows that $U_{1}=\mathbf{T} U_{1}$, with $\mathbf{T}$ given by (3.3) and $h$ given by (3.2), hence $h\left(X, U_{1}(X)\right)=-\frac{(1+o(1)) \log X}{4 X^{2}}$, hence $U_{1} \in \mathcal{E}$, hence $U_{1} \in \mathcal{B}^{\prime}(0, L)$ for some $L>0$ large enough, hence $U_{1}=U_{+}$by Lemma 3.1.

For a proof of the statement on the derivative $Y_{+}^{\prime}$, we change $K$ into $K+1$ and we use Cauchy's formula

$$
\varphi^{\prime}(X)=\frac{1}{2 \pi i} \int_{|z-X|=\sin \delta} \frac{\varphi(z)}{(z-X)^{2}} d z
$$

applied to the function

$$
\varphi: X \mapsto Y_{+}(X)-\alpha X^{1 / 2}-\frac{\alpha}{8} X^{-1 / 2} \log X
$$

Since $\varphi(z)=\mathcal{O}\left(X^{-3 / 2}(\log X)^{2}\right)$ uniformly for all $z$ such that $|z-X|=\sin \delta$, we obtain $\varphi^{\prime}(X)=\mathcal{O}\left(X^{-3 / 2}(\log X)^{2}\right)$ as well, hence the wanted estimate for $Y_{+}^{\prime}$.

Remark. Modifying $\delta$ if necessary, we can also prove that

$$
Y_{+}^{\prime}(X)=\frac{\alpha}{2} X^{-1 / 2}+\frac{\alpha}{8}\left(X^{-1 / 2} \log X\right)^{\prime}+\mathcal{O}\left(\left(X^{-3 / 2}(\log X)^{2}\right)^{\prime}\right), \Omega_{+} \ni X \rightarrow \infty .
$$

In order to prove the statements on the inverse function $V_{+}(Z)$ we show first

Lemma 3.2 . If $K>0$ is large enough, then for every $Z \in Q_{+}(2 K, 2 \delta)$ there exists a unique $X \in \Omega_{+}(K, \delta)$ such that $Y_{+}(X)=Z$.

Proof. It suffices to show that for every $U \in \Omega_{+}(2 K, 2 \delta)$ there is a unique $X \in \Omega_{+}(K, \delta)$ such that $\alpha^{-2} Y_{+}(X)^{2}=U$. By the estimate we proved above, we have

$$
\alpha^{-2} Y_{+}(X)^{2}=X+\frac{1}{4} \log X+o(1), X \rightarrow \infty .
$$

This suggests to apply Rouché's theorem to $f(X)=\alpha^{-2} Y_{+}(X)^{2}-U$ and $g(X)=X-$ $U$. Clearly $g$ has exactly one zero in $\Omega_{+}(K, \delta)$. If we show that $|f(X)-g(X)|<|g(X)|$ on the boundary of $\Omega_{+}(K, \delta)$, then the hypotheses of Rouché's theorem are satisfied and we obtain the wanted statement that $f$ has a unique zero in $\Omega_{+}(K, \delta)$. The fact that we work with infinite domains is not a problem here, because we can (for given $U$ ) add a circular arc $|X|=L,|\arg (X)| \leq \pi-\delta$ with large radius $L$ to the boundary and the condition $|f(X)-g(X)|=\left|\frac{1}{4} \log X+o(1)\right|<|X-U|=|g(X)|$ is satisfied there.

So we want to show that, if $K$ is large enough, then

$$
\left|\alpha^{-2} Y_{+}(X)^{2}-X\right|<|X-U| \text { for } U \in \Omega_{+}(2 K, 2 \delta) \text { and } X \in \partial \Omega_{+}(K, \delta) .
$$


By (3.8) and $\frac{\log X}{|X|} \rightarrow 0$ as $X \rightarrow \infty$ on $\partial \Omega_{+}(K, \delta)$, it is sufficient to show that

$$
|X-U| \geq|X| \sin \delta \text { for } U \in \Omega_{+}(2 K, 2 \delta) \text { and } X \in \partial \Omega_{+}(K, \delta),
$$

if $K$ is sufficiently large. In order to show this estimate, we consider, for every $X$ on the ray $\arg (X-K)=\pi-\delta$, its projection $U_{P}(X)$ on the ray $\arg (U-2 K)=\pi-2 \delta$. Let $C$ denote the intersection of the opposite rays $\arg (X-K)=-\delta$ and $\arg (U-2 K)=$ $-2 \delta$. Since the triangle $(K, 2 K, C)$ is isosceles at $2 K$, we have $|X|<|X-C|$ and $\left|X-U_{P}(X)\right|=|X-C| \sin \delta$ for every $X$ on the ray $\arg (X-K)=\pi-\delta$. To sum up, we have, for all $U \in \Omega_{+}(2 K, 2 \delta)$ and all $X$ with $\arg (X-K)=\pi-\delta$

$$
|X-U| \geq\left|X-U_{P}(X)\right|=|X-C| \sin \delta \geq|X| \sin \delta .
$$

By symmetry, the same inequality holds for $X$ on the other half of $\partial \Omega_{+}(K, \delta)$, i.e. $X$ with $\arg (X-K)=-\pi+\delta$, and (3.10) is finally proved.

Lemma 3.2 shows the existence of an inverse function $V_{+}: Q_{+}(2 K, 2 \delta) \rightarrow \Omega_{+}(K, \delta)$. Using a classical statement on holomorphic functions (see e.g. [6], Section 10.33) we prove that $V_{+}$is holomorphic. Since $Y_{+}(X)=\alpha X^{1 / 2}(1+o(1))$, we first obtain $V_{+}(Z)=$ $\left(\frac{Z}{\alpha}\right)^{2}(1+o(1))$ by replacing $X=V_{+}(Z)$. The estimate for $Y_{+}(X)$ yields more precisely

$$
\frac{Z}{\alpha}=V_{+}(Z)^{1 / 2}+\frac{1}{8} V_{+}(Z)^{-1 / 2} \log \left(V_{+}(Z)\right)+o\left(V_{+}(Z)^{-1 / 2}\right)
$$

and thus $V_{+}(Z)=\left(\frac{Z}{\alpha}\right)^{2}-\frac{1}{2} \log \left(\frac{Z}{\alpha}\right)+o(1)$. The functional equation for $V_{+}$follows immediately from the difference equation (2.6) of $Y_{+}$replacing $X=V_{+}(Z)$.

\section{A bounded inverse of $\Delta_{\varepsilon}$ on a bounded domain.}

Given $\delta, \varepsilon_{0}>0$ small enough, let $S=S\left(-\frac{\delta}{2}, \frac{\delta}{2}, \varepsilon_{0}\right)$ denote the sector

$$
S=\left\{\varepsilon \in \mathbb{C} ;|\arg \varepsilon|<\delta,|\varepsilon|<\varepsilon_{0}\right\} .
$$

As before, $\mu=\sin \frac{\delta}{2}$ and $\Omega_{1}$ is described in Figure 2.2. Then $\Omega_{1}$ has the following property: For all $x \in \Omega_{1}$ there exists a path $\gamma_{x}:[0,1] \rightarrow \Omega_{1} \cup\{-i r, i r, K \varepsilon\}$, joining $-i r$ and $i r$ and passing through $x$, which is $(\mu, d)$-ascending for all $d \in\left[-\frac{\delta}{2}, \frac{\delta}{2}\right]$ in the following sense: If $s<t$ then $\operatorname{Im}\left(\left(\gamma_{x}(t)-\gamma_{x}(s)\right) e^{-i d}\right) \geq \mu\left|\gamma_{x}(t)-\gamma_{x}(s)\right|$. In fact $\gamma_{x}$ can be chosen piecewise polygonal.

Assume $K \geq \frac{1}{2}$ and $\varepsilon_{0} \leq 2 r$, and let

$$
\widetilde{\Omega}=\Omega_{1}(\varepsilon)+\left[-\frac{\varepsilon}{2}, \frac{\varepsilon}{2}\right]=\left\{x+\tau ; x \in \Omega_{1}(\varepsilon),-\frac{\varepsilon}{2} \leq \tau \leq \frac{\varepsilon}{2}\right\} .
$$

Let $\mathcal{H}_{0}$ denote the space of bounded holomorphic functions on $\widetilde{\Omega}$, endowed with the supremum norm. Observe that, for all $\varepsilon \in S$ and all $x \in \widetilde{\Omega}$, we have $\frac{K}{2}|\varepsilon| \mu \leq|x| \leq 2 r$. 
Given $x_{0} \in \operatorname{cl}(\widetilde{\Omega})$, depending on $\varepsilon$ or not, let $\mathbf{S}_{x_{0}}$ denote the integration operator defined by $\mathbf{S}_{x_{0}} f(x)=\int_{x_{0}}^{x} f(t) d t$.

We reproduce below some results of [3], in particular Theorem 2 and its extension for $\varepsilon$ complex described in Section 5 of [3]. These results can be gathered in the following statement.

Proposition 4.1 . There exists a bounded linear operator $\mathbf{U}_{\varepsilon}: \mathcal{H}_{0} \rightarrow \mathcal{H}_{0}$, satisfying $\left\|\mathbf{U}_{\varepsilon}\right\| \leq \frac{5}{\mu^{2}}$, such that, for all $x_{0} \in \operatorname{cl}(\widetilde{\Omega})$, the operator $\mathbf{V}_{\varepsilon}^{0}=\mathbf{S}_{x_{0}}-\varepsilon \mathbf{U}_{\varepsilon}$ is a right inverse of $\Delta_{\varepsilon}$, i.e. we have $\Delta_{\varepsilon} \mathbf{V}_{\varepsilon}^{0} f(x)=f(x)$ for all $f \in \mathcal{H}_{0}$ and all $x \in \widetilde{\Omega} \cap(\widetilde{\Omega}-\varepsilon)$.

In the sequel we present an extension of this result for other normed spaces. Given $a \in \mathbb{R}$, let $\mathcal{H}_{a}$ denote the same space as $\mathcal{H}_{0}$ of bounded holomorphic functions in $\widetilde{\Omega}$, but endowed with the norm $\left.\|f\|_{a}:=\sup _{x \in \widetilde{\Omega}}\left|x^{-a} f(x)\right|<+\infty\right\}$. Observe that, if $a, b \in \mathbb{R}, f \in \mathcal{H}_{a}$, and $g \in \mathcal{H}_{b}$, then $f g \in \mathcal{H}_{a+b}$ and $\|f g\|_{a+b} \leq\|f\|_{a}\|g\|_{b} \|$.

Observe also that, if $a<b$ and $f \in \mathcal{H}_{b}$, then $f \in \mathcal{H}_{a}$ and

$$
\|f\|_{a} \leq \widetilde{r}^{b-a}\|f\|_{b}
$$

with $\widetilde{r}=r+\frac{\varepsilon_{0}}{2}$. As a consequence, because we can reduce $\varepsilon_{0}$ and $r$ if necessary, in a sum $f+g$ with $f \in \mathcal{H}_{a}$ and $g \in \mathcal{H}_{b}, a<b$, we will keep in mind that $g$ can be neglected, roughly speaking.

Given a bounded linear operator $F: \mathcal{H}_{a} \rightarrow \mathcal{H}_{b}$, we denote by $\|F\|_{a}^{b}$ its norm, i.e. the best constant such that

$$
\|F f\|_{b} \leq\|F\|_{a}^{b}\|f\|_{a} \text { for all } f \in \mathcal{H}_{a} .
$$

The main result of this section is the following.

Theorem 4.2 . For any $a \in \mathbb{R} \backslash\{-1\}$, there exists a linear operator $\mathbf{V}_{\varepsilon}: \mathcal{H}_{a} \rightarrow \mathcal{H}_{a+1}$ with the following properties.

(i) $\mathbf{V}_{\varepsilon}$ is a right inverse of $\Delta_{\varepsilon}$, i.e. we have $\mathbf{V}_{\varepsilon} f(x+\varepsilon)-\mathbf{V}_{\varepsilon} f(x)=\varepsilon f(x)$ for all $f \in \mathcal{H}_{a}$ and all $x \in \widetilde{\Omega} \cap(\widetilde{\Omega}-\varepsilon)$.

(ii) $\mathbf{V}_{\varepsilon}$ is bounded uniformly with respect to $\varepsilon$. More precisely, $\left\|\mathbf{V}_{\varepsilon}\right\|_{a}^{a+1}$ is bounded by a constant $L(a, K, r, \delta)$ depending only on $a, K, r$, and $\delta$.

(iii) In the case $a<-1$, we have $\mathbf{V}_{\varepsilon} f(r)=0$ for all $f \in \mathcal{H}_{a}$. In the case $a>-1$, we have $\mathbf{V}_{\varepsilon} f(K \varepsilon)=0$ for all $f \in \mathcal{H}_{a}$.

Remark. In the case $a=-1$, one cannot expect a bound independent of $\varepsilon$ for any $\mathbf{V}_{\varepsilon}: \mathcal{H}_{-1} \rightarrow \mathcal{H}_{0}$. Indeed, this would give a bound for some $\mathbf{S}_{x_{0}}$ at least on the interval $[K \varepsilon, r]$, i.e. a bound for an antiderivative of $1 / x$ independent of $\varepsilon$ on this interval, which is impossible. 
Idea of proof. Given $a \in \mathbb{R} \backslash\{-1\}$ and $h \in \mathcal{H}_{a}$, we have to solve equation $\Delta_{\varepsilon} u=h, u \in$ $\mathcal{H}_{a+1}$. In order to use Proposition 4.1, we make the change of unknown $u(x)=x^{a} v(x)$. This yields equation

$$
\Delta_{\varepsilon} v=-c_{a} v+k, v \in \mathcal{H}_{1}
$$

with

$$
c_{a}(x)=\frac{(x+\varepsilon)^{a}-x^{a}}{\varepsilon(x+\varepsilon)^{a}} \text { and } k(x)=(x+\varepsilon)^{-a} h(x) \in \mathcal{H}_{0} .
$$

We then consider the right inverse $\mathbf{V}_{\varepsilon}^{0}$ of $\Delta_{\varepsilon}$ given by Proposition 4.1 , with a choice of $x_{0}$ depending upon whether $a<-1$ or $a>-1$. Precisely, if $a<-1$, then we choose $\mathbf{V}_{\varepsilon}^{0}=\mathbf{S}_{r}-\varepsilon \mathbf{U}_{\varepsilon}$, and if $a>-1$, then we choose $\mathbf{V}_{\varepsilon}^{0}=\mathbf{S}_{K \varepsilon}-\varepsilon \mathbf{U}_{\varepsilon}$. Actually, Lemma 4.3 below says that, in both cases, $\mathbf{S}$ is bounded uniformly with respect to $\varepsilon$. The tedious and lengthy proof is omitted.

\section{Lemma 4.3 .}

(a) If $a>-1$, then $\mathbf{S}_{K \varepsilon}: \mathcal{H}_{a} \rightarrow \mathcal{H}_{a+1}$ is bounded by a constant depending only on $a$ and $\delta$.

(b) If $a<-1$, then $\mathbf{S}_{r}: \mathcal{H}_{a} \rightarrow \mathcal{H}_{a+1}$ is bounded by a constant depending only on $a$ and $\delta$.

In the sequel, $\mathbf{S}$ alone will denote either $\mathbf{S}_{r}$ or $\mathbf{S}_{K \varepsilon}$. As a consequence, a solution of equation

$$
v=\mathbf{V}_{\varepsilon}^{0}\left(k-c_{a} v\right)=\left(\mathbf{S}-\varepsilon \mathbf{U}_{\varepsilon}\right)\left(k-c_{a} v\right)
$$

will be a solution of (4.3). Passing on the left hand side the main part depending on $v$ of the right hand side, we now rewrite this latter equation in the form

$$
v+\mathbf{S}\left(c_{a} v\right)=\varepsilon \mathbf{U}_{\varepsilon}\left(c_{a} v\right)+\mathbf{V}_{\varepsilon}^{0} k .
$$

We then construct a right inverse $\mathbf{T}_{a}$ of the operator id $+\mathbf{S} c_{a}: v \mapsto v+\mathbf{S}\left(c_{a} v\right)$ which is bounded in norm by a constant independent of $\varepsilon$. Now the operator $v \mapsto$ $v-\mathbf{T}_{a}\left(\varepsilon \mathbf{U}_{\varepsilon}\left(c_{a} v\right)\right)$ from $\mathcal{H}_{1}$ to $\mathcal{H}_{1}$; is close to identity, hence has an inverse, denoted by $\mathbf{P}$. Lastly, a solution of (4.3) is given by $v=\mathbf{P} \mathbf{T}_{\varepsilon} \mathbf{V}_{\varepsilon}^{0} k$. The complete proofs will appear in a forthcoming article.

\section{Proof of Theorem 2.2.}

We prove the statement only for $y_{1}$. The symmetries imply the statement for $y_{2}$ and the proof for $y_{3}, y_{4}$ is analogous. Before the proof, we have to introduce some notation. Set $y_{+}(x)=\eta Y_{+}\left(\frac{x}{\varepsilon}\right)$; in this manner, $y_{+}$is a solution of

$$
\Delta_{\varepsilon} y_{+}=\frac{\alpha^{2}}{2 y_{+}} \text {. }
$$


By Proposition 2.1, there exists a constant $C>0$, depending only on $\delta$, such that for $K$ large enough, $\eta_{0}$ and $r$ small enough, and all $x \in \Omega_{1}$,

$$
\left|y_{+}(x)-\alpha x^{1 / 2}\right| \leq C\left|x^{-1 / 2} \varepsilon \log \frac{x}{\varepsilon}\right| \quad \text { and } \quad\left|y_{+}^{\prime}(x)-\frac{\alpha}{2} x^{-1 / 2}\right| \leq C\left|x^{-3 / 2} \varepsilon\left(\log \frac{x}{\varepsilon}\right)^{2}\right| .
$$

In particular, the functions $x \mapsto x^{-1 / 2} y_{+}(x)$ and $x \mapsto x^{1 / 2} y_{+}^{\prime}(x)$ are bounded above and below by constants independent of $\varepsilon$.

The notation $\sigma_{\varepsilon}$ stands for the shift operator given by $\sigma_{\varepsilon}(x)=x+\varepsilon$. This operator will be used in the following Leibniz-type rule:

$$
\Delta_{\varepsilon}(f g)=\left(\Delta_{\varepsilon} f\right) g+\left(f \circ \sigma_{\varepsilon}\right)\left(\Delta_{\varepsilon} g\right)
$$

Let $C_{j}=C_{j}(\varepsilon)$ denote the constants

$$
C_{1}=\left\|y_{+}^{\prime}\right\|_{-1 / 2} \quad \text { and } \quad C_{2}=\left\|1 /\left(y_{+}^{\prime} \circ \sigma_{\varepsilon}\right)\right\|_{1 / 2} .
$$

Given $a \in \mathbb{R} \backslash\{-1\}$ and $f \in \mathcal{H}_{a}$ and $r>0$, the closed ball of center $f$ and radius $r$ is denoted by $\mathcal{B}_{a}^{\prime}(f, r)$, and $\mathcal{B}$ is the closed ball

$$
\mathcal{B}=\mathcal{B}_{1 / 2}^{\prime}\left(y_{+},\left\|\frac{y_{+}}{2}\right\|_{1 / 2}\right) \subset \mathcal{H}_{1 / 2},
$$

The function $g$ is defined by

$$
g(0)=f^{\prime}(0) \text { and } g(y)=\frac{1}{y}(f(y)-f(0)) \text { for } y \neq 0 .
$$

Our last notations are

$$
\begin{gathered}
G=\sup _{y \in \mathcal{B}_{+}^{\prime}}\|g(y)\|_{0} \quad \text { and } \quad G^{\prime}=\sup _{y \in \mathcal{B}_{+}^{\prime}}\left\|g^{\prime}(y)\right\|_{0}, \\
R=2 C_{1} C_{2}\left\|\mathbf{V}_{\varepsilon}\right\|_{1 / 2}^{3 / 2} G \quad \text { and } \quad r_{0}=\left(\frac{\left\|y_{+}\right\|_{1 / 2}}{2 R}\right)^{2}
\end{gathered}
$$

with the notation of (4.2), and

$$
\mathcal{B}_{R}=\mathcal{B}_{1}^{\prime}(0, R) \subset \mathcal{H}_{1} .
$$

Reducing if needed the constants $\varepsilon_{0}$ and $r$ which define $S$ and $\Omega_{1}$, we assume that $\widetilde{r}=$ $r+\frac{\varepsilon_{0}}{2} \leq r_{0}$. In this manner, for all $u \in \mathcal{B}_{R} \subset \mathcal{H}_{1}$, we have $u \in \mathcal{H}_{1 / 2}$ and

$$
\|u\|_{1 / 2} \leq \widetilde{r}^{1 / 2}\|u\|_{1} \leq \widetilde{r}^{1 / 2} R \leq r_{0}^{1 / 2} R=\left\|\frac{y_{+}}{2}\right\|_{1 / 2},
$$

hence $y_{+}+u \in \mathcal{B}$. 
Let us now begin the proof. The change of unknown $y_{1}=y_{+}+u$ yields $\Delta_{\varepsilon} y_{+}+$ $\Delta_{\varepsilon} u=\frac{1}{y_{+}+u} f\left(y_{+}+u\right)$. Using (5.1) and using $g$ given by (5.5), we obtain

$$
\Delta_{\varepsilon} u=\frac{\alpha^{2}}{2\left(y_{+}+u\right)}-\frac{\alpha^{2}}{2 y_{+}}+g\left(y_{+}+u\right)=-\frac{-\alpha^{2} u}{2\left(y_{+}+u\right) y_{+}}+g\left(y_{+}+u\right) .
$$

We rewrite this equation as follows

$$
\Delta_{\varepsilon} u=-\frac{\alpha^{2} u}{2 y_{+}^{2}}+\frac{\alpha^{2} u^{2}}{2\left(y_{+}+u\right) y_{+}^{2}}+g\left(y_{+}+u\right) .
$$

In a first time, we consider the following linear equation

$$
\Delta_{\varepsilon} u=-\frac{\alpha^{2} u}{2 y_{+}^{2}}+k
$$

In order to solve (5.10), first observe that the derivative $y_{+}^{\prime}$ is a solution of the associated homogeneous equation. Indeed, differentiating (5.1) yields $\Delta_{\varepsilon} y_{+}^{\prime}=-\frac{\alpha^{2} y_{+}^{\prime}}{2 y_{+}^{2}}$. We then use the method of variation of constant, i.e. the change $u=y_{+}^{\prime} v$. Since

$$
\Delta_{\varepsilon} u=\left(\Delta_{\varepsilon} y_{+}^{\prime}\right) v+\left(y_{+}^{\prime} \circ \sigma_{\varepsilon}\right) \Delta_{\varepsilon} v=-\frac{\alpha^{2} y_{+}^{\prime}}{2 y_{+}^{2}} v+\left(y_{+}^{\prime} \circ \sigma_{\varepsilon}\right) \Delta_{\varepsilon} v,
$$

equation (5.10) yields for $v$ the equation $\Delta_{\varepsilon} v=\frac{k}{y_{+}^{\prime} \circ \sigma_{\varepsilon}}$. This latter equation can be solved using the operator $\mathbf{V}_{\varepsilon}$ given by Theorem 4.2.

We therefore consider the operator $\mathbf{T}_{\varepsilon}: \mathcal{H}_{0} \rightarrow \mathcal{H}_{1}$ given by

$$
\mathbf{T}_{\varepsilon} k=y_{+}^{\prime} \cdot \mathbf{V}_{\varepsilon}\left(\frac{k}{y_{+}^{\prime} \circ \sigma_{\varepsilon}}\right) .
$$

To sum up, the operator $\mathbf{T}_{\varepsilon}$ solves (5.10), i.e. $u=\mathbf{T}_{\varepsilon} k$ is a solution of this equation.

Lemma 5.1 . The operator $\mathbf{T}_{\varepsilon}: \mathcal{H}_{0} \rightarrow \mathcal{H}_{1}$ is bounded uniformly with respect to $\varepsilon$. Precisely, we have

with $C_{1}, C_{2}$ given by (5.3).

$$
\left\|\mathbf{T}_{\varepsilon}\right\|_{0}^{1} \leq C_{1} C_{2}\left\|\mathbf{V}_{\varepsilon}\right\|_{1 / 2}^{3 / 2}
$$

Proof. Let $k \in \mathcal{H}_{0}$; then $\frac{k}{y_{+}^{\prime} \circ \sigma_{\varepsilon}} \in \mathcal{H}_{1 / 2}$, hence $\mathbf{V}_{\varepsilon}\left(\frac{k}{y_{+}^{\prime} \circ \sigma_{\varepsilon}}\right) \in \mathcal{H}_{3 / 2}$, hence $\mathbf{T}_{\varepsilon} k \in \mathcal{H}_{1}$, and

$$
\left\|\mathbf{T}_{\varepsilon} k\right\|_{1} \leq C_{1}\left\|\mathbf{V}_{\varepsilon}\left(\frac{k}{y_{+}^{\prime} \circ \sigma_{\varepsilon}}\right)\right\|_{3 / 2} \leq C_{1}\left\|\mathbf{V}_{\varepsilon}\right\|_{1 / 2}^{3 / 2}\left\|\frac{k}{y_{+}^{\prime} \circ \sigma_{\varepsilon}}\right\|_{1 / 2} \leq C_{1} C_{2}\left\|\mathbf{V}_{\varepsilon}\right\|_{1 / 2}^{3 / 2}\|k\|_{0}
$$

Let us now return to equation (5.9). Recall that $\mathcal{B}_{R}$ is defined in (5.8). 
Lemma 5.2 . If $r>0$ and $\varepsilon_{0}>0$ are small enough and $K$ is large enough then, for all $\varepsilon \in] 0, \varepsilon_{0}[$, the map

$$
\mathbf{M}_{\varepsilon}: \mathcal{B}_{R} \rightarrow \mathcal{B}_{R}, u \mapsto \mathbf{T}_{\varepsilon}\left(\frac{\alpha^{2} u^{2}}{2\left(y_{+}+u\right) y_{+}^{2}}+g\left(y_{+}+u\right)\right)
$$

is a contraction.

Proof. Let $u \in \mathcal{B}_{R}$ and $r, \varepsilon_{0}$ be such that $\widetilde{r}=r+\frac{\varepsilon_{0}}{2} \leq r_{0}$. We have $y_{+}+u \in \mathcal{B}$, hence $\left\|g\left(y_{+}+u\right)\right\|_{0} \leq G$. We also have $\frac{\alpha^{2} u^{2}}{2\left(y_{+}+u\right) y_{+}^{2}} \in \mathcal{H}_{1 / 2}$ and

$$
\left\|\frac{\alpha^{2} u^{2}}{2\left(y_{+}+u\right) y_{+}^{2}}\right\|_{1 / 2} \leq \alpha^{2}\left(\|u\|_{1}\right)^{2}\left(\left\|\frac{1}{y_{+}}\right\|_{1 / 2}\right)^{3} \leq \alpha^{2} R^{2}\left(\left\|\frac{1}{y_{+}}\right\|_{1 / 2}\right)^{3},
$$

hence, by (4.1),

$$
\left\|\frac{\alpha^{2} u^{2}}{2\left(y_{+}+u\right) y_{+}^{2}}\right\|_{0} \leq\left\|\frac{\alpha^{2} u^{2}}{2\left(y_{+}+u\right) y_{+}^{2}}\right\|_{1 / 2} \widetilde{r}^{1 / 2} \leq G \text { if } \widetilde{r} \leq G^{2}\left(\alpha^{2} R^{2}\left(\left\|\frac{1}{y_{+}}\right\|_{1 / 2}\right)^{3}\right)^{-2}
$$

Since $R=2 C_{1} C_{2}\left\|\mathbf{V}_{\varepsilon}\right\|_{1 / 2}^{3 / 2} G \geq\left\|\mathbf{T}_{\varepsilon}\right\|_{0}^{1} G$, this proves that $\mathbf{M}_{\varepsilon}(u) \in \mathcal{B}_{R}$. We prove similarly that $\mathbf{M}_{\varepsilon}$ is a contraction.

To conclude, the unique fixed point $u^{*}$ of $\mathbf{M}_{\varepsilon}$ in $\mathcal{B}_{R}$ is a solution of (5.9). Moreover, since we are in the case $a=\frac{1}{2}>-1$ of Theorem 4.2(iii), we have $u^{*}(K \varepsilon)=0$. Therefore the function $y_{1}=y_{+}+u^{*}$ satisfies the conditions of Theorem 2.2.

\section{Fatou coordinates: Proof of Theorem 2.4.}

We begin this section with some auxiliary results, which are useful not only for this section but also for Section 7. The proofs are straightforward but the details are a bit cumbersome.

To simplify notation, we do not indicate the $\varepsilon$-dependence of most functions. At some instances during the proofs, the domains must be reduced slightly, for example to allow a derivative of a bounded function to still be bounded. For the sake of simplicity, we will also not indicate this here.

Lemma 6.1 . For $j=1,2$, let $y_{j}: D_{j} \rightarrow \mathbb{C}$ be solutions of (2.1) on domains $D_{j}$ not containing 0 . We suppose that $y_{j}(x)=y_{0}(x)+\mathcal{O}(\varepsilon)$ uniformly for $x \in D_{j}$ and that $D_{1} \cap D_{2}$ is connected. Let $b^{+}$, resp. $b^{-} \in \mathbb{R}$ denote the maximum, resp. minimum, of $\operatorname{Im} x$ on $D_{1} \cap D_{2}$.

Then, for any $\delta$ small enough, there exists an $\varepsilon$-periodic function $p: S \rightarrow \mathbb{C}$ defined on the strip $S=\left\{x \in \mathbb{C} ; b^{-}+\delta<\operatorname{Im} x<b^{+}-\delta\right\}$ and satisfying $y_{2}(x)=y_{1}(x+p(x))$ for all $x \in D_{1} \cap D_{2} \cap S$. 
By Rouché's theorem, it can be proved that $y_{1}$ is locally invertible; let $v_{1}$ denote such a local inverse. The function $p$ is then simply given by $p(x)=v_{1}\left(y_{2}(x)\right)-x$. As both $y_{1}$ and $y_{2}$ are close to $y_{0}$, we have $p(x)=\mathcal{O}(\varepsilon)$. Since both satisfy (2.1), the $\varepsilon$-periodicity of $p$ follows.

Corollary 6.2 . With the notation of Lemma 6.1, let $\Omega \subseteq\left(D_{1} \cup D_{2}\right) \cap S$ be a horizontally convex domain (i.e. $x, x^{\prime} \in \Omega$ and $\operatorname{Im} x=\operatorname{Im} x^{\prime}$ imply $\left[x, x^{\prime}\right] \subset \Omega$ ). Then the solution $y_{2}$ can be analytically continued on $\Omega$ by the formula of Lemma 6.1.

Of course, $y_{2}$ is still a solution of (2.1) on $\Omega$. By symmetry, $y_{1}$ can also be analytically continued on $\Omega$ by the formula $y_{1}(x)=y_{2}(x+q(x))$ with the $\varepsilon$-periodic function $q(x)=$ $v_{2}\left(y_{1}(x)\right)-x$.

Corollary 6.3 . With the above notation, there exists a function $s=s(\varepsilon)$ such that the function $R: \Omega \rightarrow \mathbb{C}, x \mapsto y_{1}(x)-y_{2}(x+s(\varepsilon))$ is exponentially small. More precisely, if $d(x)=\min \left(\operatorname{Im} x-b^{-}+\delta, b^{+}-\operatorname{Im} x-\delta\right)$, then we have $R(x)=\mathcal{O}\left(e^{-2 \pi d(x) / \varepsilon}\right)$.

The function $s$ is simply the constant term $c_{0}$ in the Fourier expansion of $p$

$$
p(x)=\sum_{\nu \in \mathbb{Z}} c_{\nu} e^{2 \pi i \nu x / \varepsilon} .
$$

The function $s$ is called the shift in Section 7. The next result is based on general results of [3].

Corollary 6.4 . Let $D_{1} \subset D_{2}$ be horizontally convex domains. Assume that there exists a solution $y_{1}: D_{1} \rightarrow \mathbb{C}$ of (2.1) and that the solution $y_{0}=a_{0}^{-1}$ of (2.2) is defined on $D_{2}$. Let $b^{+}$, resp. $b^{-} \in \mathbb{R}$ denote the maximum, resp. minimum, of $\operatorname{Im} x$ on $D_{1}$.

Then, for any compact subset $K$ of $D_{2}$ and any $\delta>0$, there exists $\varepsilon_{0}>0$ such that, for all $\left.\varepsilon \in] 0, \varepsilon_{0}\right], y_{1}$ can be analytically continued onto $K \cap S$, with

$$
S=\left\{x \in \mathbb{C} ; b^{-}+\delta<\operatorname{Im} x<b^{+}-\delta\right\} .
$$

Actually, by Theorem 7 of [3], there exists a solution $y_{2}$ on $K$. Therefore, by Corollary 6.2 above, $y_{1}$ can be continued on $K \cap S$.

Proof of Theorem 2.4. A consequence of Proposition 2.3 and of the estimate for $V_{+}$in Proposition 2.1 is that, if $\gamma>0$ arbitrarily small is fixed, then for $r$ small enough and $K$ large enough, the function $v_{1}$ satisfies

$$
\forall u \in \widetilde{Q}_{1}, \quad(1-\gamma)\left|\frac{u}{\alpha}\right|^{2} \leq\left|v_{1}(u)\right| \leq(1+\gamma)\left|\frac{u}{\alpha}\right|^{2} .
$$

Now we consider other arguments for $\eta$ (and thus of $\varepsilon=\eta^{2}$ ). It can be shown that Theorem 2.2 and Proposition 2.3 are also valid if the interval $] 0, \varepsilon]$ is replaced by a sector with sufficiently small opening angle bisected by the positive real axis. 
Then let $\left(S_{l}\right)_{l=1}^{L}$ be a good covering of the origin (in the $\eta$-plane) by sectors of opening at most $2 \delta$. Since each $\eta$-sector $S_{l}$ can be reduced to a sector bisected by the positive real axis using a rotation, the previous results can be carried over to $S_{l}$. As such a rotation changes $\alpha$ to $\exp (2 \pi i l / L) \alpha$, this leads to functions $v_{l}^{j}, j=1, \ldots, 4$ on domains $Q_{l}^{j}=$ $\exp (2 \pi i l / L) Q_{j}, Q_{j}$ defined above Theorem 2.2, that are analogous to the functions of Proposition 2.3; especially they satisfy (2.11) and are inverse to solutions $y_{l}^{j}$ of (2.1).

Next we show that, on the intersections $Q_{l}^{j} \cap Q_{l+1}^{j}$, we have

$$
\left|\left(v_{l+1}^{j}\right)^{\prime}(z)-\left(v_{l}^{j}\right)^{\prime}(z)\right| \leq K \exp \left(-\frac{\alpha}{|\eta|^{2}}\right)
$$

whereas, on the intersections $Q_{l}^{j} \cap Q_{l}^{j+1}$, we have

$$
\left|\left(v_{l}^{j+1}\right)^{\prime}(z)-\left(v_{l}^{j}\right)^{\prime}(z, \varepsilon)\right| \leq K|\eta| \exp \left(-\alpha\left|\frac{z}{\eta}\right|^{2}\right)
$$

with some positive constants $K, \alpha$.

For the proof, fix $j, l$. Applying Corollary 6.3 to $y_{l}^{j}$ and $y_{l+1}^{j}$, we obtain the existence of some function $s=s(\varepsilon)$ such that $y_{l}^{j}(x)-y_{l+1}^{j}(x+s(\varepsilon))$ is $\mathcal{O}\left(e^{-\alpha /|\varepsilon|}\right)$ on the intersection of their domains, with some constant $\alpha$. This implies that $\left(v_{l+1}^{j}-v_{l}^{j}\right)(z)-$ $s(\varepsilon)$ is also $\mathcal{O}\left(e^{-\alpha /|\varepsilon|}\right)$ on $Q_{l}^{j} \cap Q_{l+1}^{j}$. Now we obtain (6.2) by differentiation.

For the proof of (6.3), we have to refine Corollary 6.3 and its proof for $y_{l}^{j}$ and $y_{l}^{j+1}$. The function $p$ defined by $p(x)=\left(v_{l}^{j+1} \circ y_{l}^{j}\right)(x)-x$ is $\varepsilon$-periodic and bounded on some strip one boundary of which passes at a distance of $K|\varepsilon|$ from the origin. Using the Fourier series for $p$, its constant term $c_{0}$ and estimates for the other coefficients, we prove that $p(x)-c_{0}(\varepsilon)=\mathcal{O}\left(|\varepsilon| e^{-\mu|x| /|\varepsilon|}\right)$ with some positive $\mu$. The factor $\varepsilon$ comes from the estimate for $v_{l}^{j}$ near the origin and the corresponding estimates for Fourier coefficients.

Carrying this over to $v_{l}^{j+1}$ and $v_{l}^{j}$, we obtain that

$$
\left(v_{l}^{j+1}-v_{l}^{j}\right)(z)-c_{0}(\varepsilon)=\mathcal{O}\left(|\varepsilon| e^{-\widetilde{\mu}|z|^{2} /|\varepsilon|}\right)
$$

with some positive constant $\widetilde{\mu}$. Here some estimate analogous to (6.1) has been used. Differentiation yields $\left(v_{l}^{j+1}-v_{l}^{j}\right)^{\prime}(z)=\mathcal{O}\left(|z| e^{-\widetilde{\mu}|z|^{2} /|\varepsilon|}\right)$. This finally gives (6.3) for any positive $\alpha<\widetilde{\mu}$.

The estimates (6.2) and (6.3) are exactly the important hypotheses of the Main Theorem 4.1 of the memoir [4]. We obtain composite asymptotic expansions (CASEs) of Gevrey order 1 for the functions $w_{l}^{j}=\left(v_{l}^{j}\right)^{\prime}$. Especially, we obtain CAsEs for $v_{j}^{\prime}=\left(v_{0}^{j}\right)^{\prime}$ : 3

$$
v_{j}^{\prime}(z, \varepsilon) \sim_{\frac{1}{2}} \sum_{n=0}^{\infty}\left(A_{n}(z)+B_{n}^{j}\left(\frac{z}{\eta}\right)\right) \eta^{n}
$$

3. Starting here, we have to indicate the dependence of functions on $\varepsilon$ again. 
where the functions $A_{n}$ are holomorphic on some disk centered at the origin, $B_{n}^{j}$ are holomorphic on $i^{j-1} Q_{+}(2 K, 2 \delta)$ and have consistent asymptotic expansions of Gevrey order $\frac{1}{2}$

$$
B_{n}^{j}(Z) \sim_{\frac{1}{2}} \sum_{m \geq 1} D_{n m} Z^{-m} \text { as } Z \rightarrow \infty .
$$

We refer to the explanations below Theorem 2.4 for details.

Finally, we use the initial conditions for $v_{j}$. In the case $j=1$ (the others are analogous), we have

$$
v_{1}(z, \varepsilon)=K \varepsilon+\int_{\eta Y_{+}(K)}^{z} v_{1}^{\prime}(\zeta, \varepsilon) d \zeta .
$$

Now we separate the leading term of each $B_{n}^{1}$, i.e. we write $B_{n}^{1}(Z)=D_{n 1} Z^{-1}+C_{n}^{1}(Z)$, $C_{n}^{1}(Z)=\mathcal{O}\left(Z^{-2}\right)$ and integrate (6.4) term by term (for details see [4]). We use $a_{n}(z)=$ $\int_{0}^{z} A_{n}(\zeta) d \zeta, b_{n}^{1}(Z)=\int_{\infty}^{Z} C_{n}^{1}(u) d u$ and we collect the terms independent of $z$ in $T_{j}$. Thus we finally obtain the wanted CAsE for $v_{1}$.

The statement on $b_{0}^{j}$ and $b_{0}^{j}$ follows from the factor $\eta$ in (6.3): Theorem 4.1 of [4] applies to the family $\frac{1}{\eta}\left(v_{l}^{j}\right)^{\prime}, j=1, \ldots, 4, l=1 \ldots, L$. The leading term $a_{0}(z)$ can be determined using the Schröder equation (7.4). The fact that the right hand side of the outer expansion (2.14) is a formal solution of (7.4) implies that it contains only powers of $\varepsilon=\eta^{2}$.

\section{Application.}

We present in this section an informal study of equation (1.9), rewritten below for convenience:

$$
\Delta_{\varepsilon} y=1+\frac{1}{y}
$$

It is well known that, for fixed $\varepsilon>0$, the difference equation (7.1) has solutions holomorphic on sectors with vertex at infinity. The dependence on $\varepsilon$, however, is not clear. We start our study with the subsequent proposition. We use the notation of the somewhat similar study of the inner reduced equation of Section 3. In particular $\Omega_{+}(K, \delta)$ is defined in (2.7) and shown on Figure 2.1.

Proposition 7.1. Fix $\varepsilon_{0}>0$. For all $\delta>0$ there exists $K>0$ such that (7.1) has a unique solution $y_{+}^{\infty}$ defined for $\left.\left.\varepsilon \in\right] 0, \varepsilon_{0}\right], x \in \Omega_{+}(K, \delta)$ holomorphic with respect to $x$ satisfying

$$
y_{+}^{\infty}(x, \varepsilon)=x+\log x+o(1) \text { as } x \rightarrow \infty \text { in } \Omega_{+}(K, \delta) .
$$

Similarly, there is a unique solution $y_{-}^{\infty}$ on $-\Omega_{+}(K, \delta)$ satisfying $y_{-}^{\infty}(x, \varepsilon)=x+\log x+$ $o(1)$ as $x \rightarrow \infty$ in $-\Omega_{+}(K, \delta)$. On $-\Omega_{+}(K, \delta)$ we use the branch of the logarithm given by $\log x=\log (-x)+\pi i$; on $\Omega_{+}(K, \delta)$ we use the principal value. 
The proof is similar to that of Proposition 2.1 and is omitted. If $K$ is sufficiently large, then the solutions $y_{ \pm}^{\infty}$ have inverse functions $v_{ \pm}^{\infty}$ also called Fatou coordinates. These are holomorphic functions of their first variable in some domain containing infinite sectors $\pm \Omega_{+}(\widetilde{K}, \widetilde{\delta})$ with some $\widetilde{K}>K, \widetilde{\delta}>\delta$. They satisfy

$$
v_{ \pm}^{\infty}(z, \varepsilon)=z-\log z+o(1) \text { as } z \rightarrow \infty
$$

and the functional equation

$$
v\left(z+\varepsilon\left(1+\frac{1}{z}\right)\right)=v(z)+\varepsilon .
$$

The formula $v_{-}^{\infty} \circ y_{+}^{\infty}-$ id defines two functions $p_{ \pm}^{\infty}$ on the sector $\mathcal{I}_{+}$introduced above (2.10), respectively on $\mathcal{I}_{-}=-\mathcal{I}_{+}$. They are bounded and $\varepsilon$-periodic and hence there exist Fourier expansions

$$
p_{ \pm}^{\infty}(x, \varepsilon)=\sum_{n=0}^{\infty} c_{n \pm}^{\infty} e^{2 \pi i n x / \varepsilon}
$$

with functions $\left.\left.c_{n \pm}^{\infty}:\right] 0, \varepsilon_{0}\right] \rightarrow \mathbb{C}$ which we call Écalle-Voronin invariants of (7.1) at $\infty$. The choice of the branches of the logarithms in Proposition 7.1 implies that $c_{0+}^{\infty}=0$ and $c_{0-}^{\infty}=-2 \pi i$.

We want to study the relation between these invariants of (7.1) at infinity and its Écalle-Voronin invariants near 0 introduced above Corollary 2.5 which will be denoted by $c_{j n}^{0}(\varepsilon)$.

To this purpose, we first prove that $v_{+}^{\infty}$ can be continued up to the domain $\widetilde{Q}_{1}$ of our local solution $v_{1}^{*}$ given by Proposition 2.3 and by (2.16). The outer reduced equation of (7.1) is $y^{\prime}=1+\frac{1}{y}$, whose solutions are implicitely given by

$$
y-\log (1+y)=x+C .
$$

Then Corollary 6.4 shows that $y_{+}^{\infty}$ can be continued along the level lines $a_{0}(y)=y-$ $\log (1+y)=t+C i, t \in\left[t_{1}, t_{2}\right]$, for any $t_{1}, t_{2}, C \in \mathbb{R}, t_{1}<t_{2}, C \neq 0$. As a consequence, $v_{+}^{\infty}$ can be continued analytically onto any compact set included in the dark region displayed on Figure 7.1 top right, where $a_{0}$ is locally invertible. In particular $v_{+}^{\infty}$ can be continued on the set $\left\{z \in \widetilde{Q}_{1} ;|z|>r_{1}\right\}$ for an arbitrary $\left.r_{1} \in\right] 0, r[$. We then apply Lemma 6.1 to $y_{1}$ and $y_{+}^{\infty}$. This allows to continue $v_{+}^{\infty}$ on $\widetilde{Q}_{1}$ in its whole. By Corollary 6.3, there exists $s=s(\varepsilon)=\mathcal{O}(\varepsilon)$ such that the function $v_{+}^{\infty}-v_{1}^{*}-s(\varepsilon)$ is exponentially small in any compact subset of $\widetilde{Q}_{1}$. We call this function $s$ the shift in the sequel. We will now compare some asymptotic expansions of $v_{1}^{*}$ and $v_{+}^{\infty}$.

Therefore, we first indicate how to prove that $v_{+}^{\infty}$ does have an asymptotic expansion. For this, we consider all arguments of $\varepsilon$. Using (7.5), we prove that $\left(v_{+}^{\infty}\right)^{\prime}$ and $\left(v_{-}^{\infty}\right)^{\prime}$ are exponentially close one to each other on $\mathcal{I}_{+}$and $\mathcal{I}_{-}$, and then we apply Ramis-Sibuya's theorem (classical, see for example [4], Lemma 4.4). 

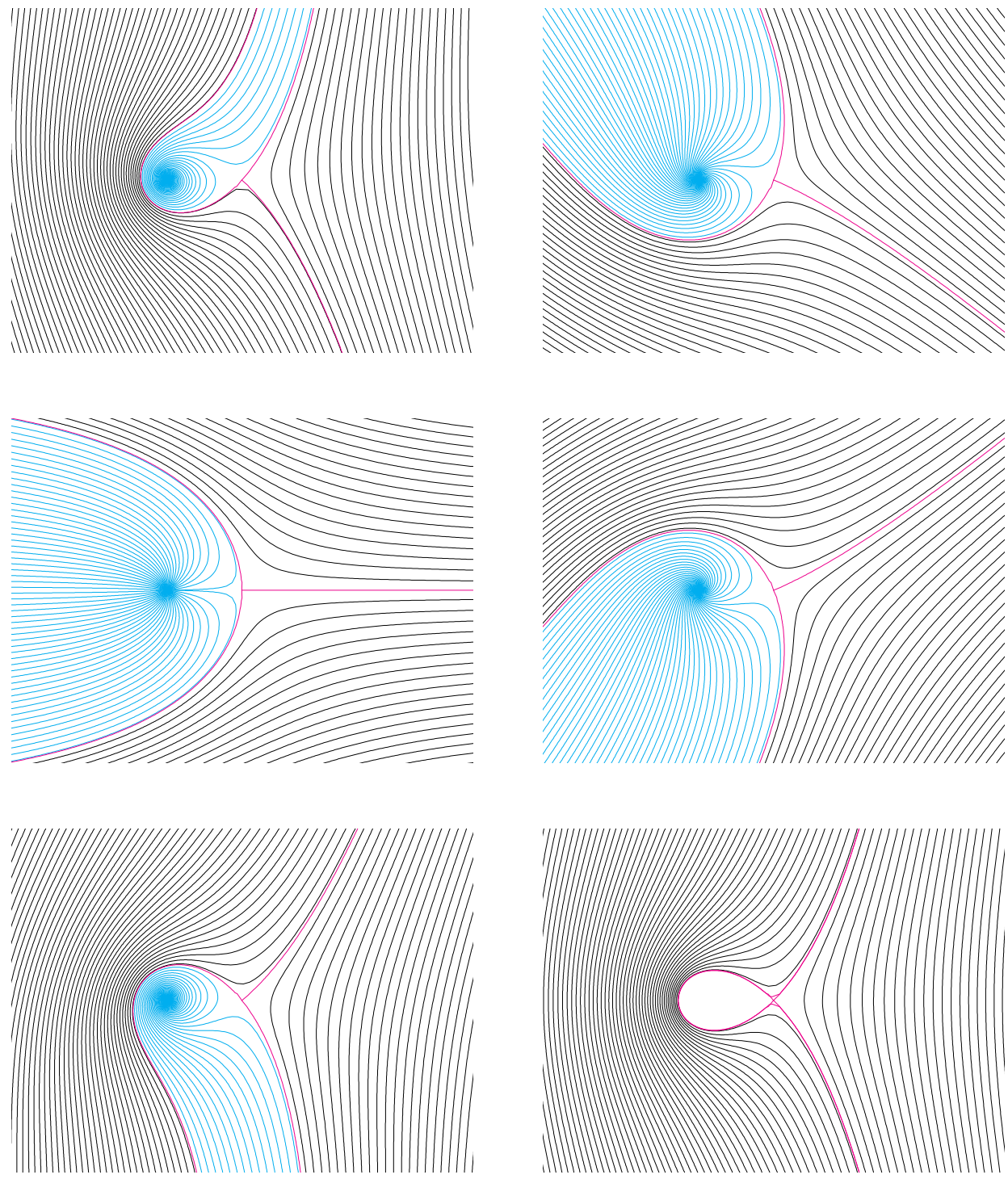

Figure 7.1. In dark, the level lines $y-\log (1+y)=(t+C) e^{i \theta}, t \in \mathbb{R}$, in the regions $D_{\theta}$, sucessively for $\theta=-\frac{\pi}{2}+0.1,-\frac{\pi}{4}, 0, \frac{\pi}{4}, \frac{\pi}{2}-0.2, \frac{\pi}{2}$.

In this manner, we obtain that $\left(v_{+}^{\infty}\right)^{\prime}$ and $\left(v_{-}^{\infty}\right)^{\prime}$ have a common asymptotic expansion $\widehat{w}(z, \varepsilon)=\sum_{n \geq 0} w_{n}(z) \varepsilon^{n}$ which is of Gevrey order 1, uniformly for $z \in Q_{+}(2 K, 2 \delta)$, 
resp. $z \in Q_{-}(2 K, 2 \delta)=i Q_{+}(2 K, 2 \delta)$. By integration, we finally obtain the desired expansion for $v_{+}^{\infty}$.

We can precisely describe $\widehat{w}$ : Using the Schröder equation (7.4), expanding its left hand side by the Taylor formula, and cancelling the terms $v(z)$, we obtain for $\widehat{w}$ the equation

$$
\sum_{n \geq 0} \frac{\varepsilon^{n}}{(n+1) !}\left(1+\frac{1}{z}\right)^{n} \widehat{w}^{(n)}(z, \varepsilon)=1-\frac{1}{1+z},
$$

from which $w_{n}$ can be determined recursively. Since, for any rational function $f,(1+$ $\left.\frac{1}{z}\right)^{n} f^{(n)}(z)$ has the same valuation in $1+z$ as $f$, we obtain that $(1+z) w_{n}(z)$ is a polynomial in $\frac{1}{z}$. More precisely, we obtain with some constants $w_{n \nu}$

$$
w_{n}(z)=\frac{1}{1+z} \sum_{\nu=n}^{2 n-1} w_{n \nu} z^{-\nu}
$$

Using a partial fraction expansion and integrating, we obtain for $v_{+}^{\infty}$ an expansion of the form

$$
v_{+}^{\infty}(z, \varepsilon) \sim_{1} z-q(\varepsilon) \log (1+z)+(q(\varepsilon)-1) \log z+\sum_{n \geq 1} v_{n}(z) \varepsilon^{n}+C(\varepsilon)
$$

where $C(\varepsilon), q(\varepsilon)$ are formal series in $\varepsilon$, and $v_{n}$ is a polynomial of degree at most $2 n-2$ in $\frac{1}{z}$ without constant term, i.e. $v_{n}(z)=\sum_{\nu=1}^{2 n-2} v_{n \nu} z^{-\nu}$.

The estimate (7.3) yields $C=0$. The Schröder equation permits also to determine $q$ explicitely. Actually, the right hand side, denoted by $\widehat{v}$, of (7.8) can be rewritten in the form

$$
\widehat{v}(z, \varepsilon)=-q(\varepsilon) \log (1+z)+\sum_{n \geq 0} h_{n}(z) \varepsilon^{n},
$$

where the functions $h_{n}$ are holomorphic in a neighborhood of $z=-1$. Since $\widehat{v}$ is a formal solution of (7.4), we obtain

$$
-q(\varepsilon) \log \left(1+\frac{\varepsilon}{z}\right)=\varepsilon+\sum_{n \geq 0}\left(h_{n}(z)-h_{n}\left(z+\varepsilon\left(1+\frac{1}{z}\right)\right)\right) \varepsilon^{n} .
$$

For the value $z=-1$, this gives

$$
q(\varepsilon)=\frac{-\varepsilon}{\log (1-\varepsilon)}
$$

We will use later on $q(\varepsilon)=1-\frac{\varepsilon}{2}+\mathcal{O}\left(\varepsilon^{2}\right)$. We want to compare the former expansion (7.8) with the outer expansion of $v_{1}^{*}$. In our example, the outer expansion (2.14) rewritten for $v_{1}^{*}=v_{1}-T_{1}$ becomes

$$
v_{1}^{*}\left(z, \eta^{2}\right) \sim_{\frac{1}{2}} S(\eta) \log \left(\frac{z}{\sqrt{2} \eta}\right)+\sum_{n \geq 0} d_{n}(z) \eta^{n}
$$


with $d_{n}(z)=a_{n}(z)+\sum_{m=1}^{n-2} B_{n-m, m} z^{-m}, a_{n}$ holomorphic in a neighborhood of 0 . Since $a_{n}(0)=0$ by Theorem 2.4, $d_{n}$ has no constant term. Now a comparison of (7.8) and (7.10) gives (with $\varepsilon=\eta^{2}$ )

$$
S(\eta)=q(\varepsilon)-1, \quad d_{n}=a_{v}+v_{n}, \quad \text { and } a_{n}=\alpha_{n} \log (1+z)
$$

with some constant $\alpha_{n}$. Concerning the shift $s$, we obtain

$$
s(\varepsilon)=S(\eta) \log (\sqrt{2} \eta)+\mathcal{O}\left(e^{-c / \varepsilon}\right)=\frac{1}{2}(q(\varepsilon)-1) \log (2 \varepsilon)+\mathcal{O}\left(e^{-c / \varepsilon}\right) .
$$

In the same manner, we can continue $v_{-}^{\infty}$ onto $\widetilde{Q}_{2}$ and compare the expansions of $v_{-}^{\infty}$ and of the local solution $v_{2}^{*}$. Regarding the Écalle-Voronin invariants $c_{n+}^{\infty}$, we finally obtain in a similar way as for (2.17) first $p_{+}^{\infty}(x, \varepsilon)=p_{1}(x-s(\varepsilon), \varepsilon)$ where $p_{1}=v_{2}^{*} \circ\left(v_{1}^{*}\right)^{-1}-$ id and thus

$$
c_{n+}^{\infty}(\varepsilon)=c_{1 n}^{0}(\varepsilon) \exp \left(-n \pi i \frac{q(\varepsilon)-1}{\varepsilon} \log (2 \varepsilon)\right) .
$$

In this manner, we obtain an asymptotic expansion of Gevrey order 1 for $c_{n+}^{\infty}$ :

$$
c_{n+}^{\infty}(\varepsilon) \exp \left(n \pi i \frac{q(\varepsilon)-1}{\varepsilon} \log (2 \varepsilon)\right) \sim_{\frac{1}{2}} \sum_{n \geq 2} a_{1 n k} \varepsilon^{k / 2} .
$$

A careful analysis shows that the former discussion can be extended to all arguments of $\varepsilon$, except $\arg \varepsilon=\frac{\pi}{2}$. In other words, for any $\delta>0$, the expansion (7.11) is valid uniformly for $\varepsilon \in S\left(-\frac{3 \pi}{2}+\delta, \frac{\pi}{2}-\delta, \varepsilon_{0}\right)$.

As shows the picture on bottom right of Figure 7.1, which corresponds to $\arg \varepsilon=\frac{\pi}{2}$, something happens for this value: There is a loop surrounding -1 that is parametrized by $y-\log (1+y)=-i t, 0<t<2 \pi$ real, where $y \sim(2 t)^{1 / 2} e^{3 \pi / 4}$ for small $t$. This makes it impossible to continue $v_{+}^{\infty}$ and $v_{-}^{\infty}$ analytically to a common set of points at a distance $\mathcal{O}(\eta)$ from the origin on the 'left hand' side of the origin (on the right hand side, there is no problem - this correponds to $\arg \varepsilon=-\frac{\pi}{2}$ ). Therefore we need an intermediate solution $v_{\alpha}$ of Schröder's equation (7.4) defined in a neighborhood of the above loop.

In order to construct such a solution, recall that the solutions of the outer reduced equation of (7.1) are given implicitly by (7.6). We are interested in the solution $y_{0}$ that parametrizes the above loop for $x \in]-2 \pi i, 0$. It satisfies $y_{0}(x)-\log \left(1+y_{0}(x)\right)=x$ and $y_{0}(x) \sim-\sqrt{2 x}$ for small $x, \arg x$ close to $-\frac{\pi}{2}$. It can be continued analytically to some domain containing the open segment $]-2 \pi i, 0[$, for example some open rhombus $G$ with vertices $0,-\beta-i \pi,-2 \pi i, \beta-i \pi$. By Theorem 7 of [3], for every compact subset $K$ of $G$ there exists a holomorphic solution $y_{\alpha}$ of (7.1) defined in some neighborhood of $K$ satisfying $y_{\alpha}(x)=y_{0}(x)+\mathcal{O}(\varepsilon)$. We choose $K$ as a rhombus with vertices $-\delta i,-\widetilde{\beta}-$ $\pi i,(\delta-2 \pi) i, \widetilde{\beta}-i \pi, \delta>0,0<\widetilde{\beta}<\beta$.

For every small $\delta>0$, there is a (also small) $\widetilde{\beta}>0$ such that $y_{0}$ is injective on $K$. As $y_{\alpha}$ is a holomorphic function close to $y_{0}$, this is also true for $y_{\alpha}$ if $\varepsilon$ is sufficiently small. 
Thus $y_{\alpha}$ has an inverse function $v_{\alpha}$ that must be a solution of (7.4) and is close to $a_{0}$ given by $a_{0}(z)=z-\log (1+z)$ on $y_{\alpha}(K)=: M$. Observe that $M$ contains no reals close to 0 because of the injectivity. For convenience, let $M_{ \pm}=\{z \in M ; \pm \operatorname{Im} z>0\}$. Observe that here $\log (1+z)$ is close to 0 if $z \in M_{+}$is small, whereas it is close to $2 \pi i$ if $z \in M_{-}$ is small.

Near $z=0$, four solutions of (7.4) can be constructed as indicated in Section 6, i.e. analogously to Proposition 2.3. Let $v_{j}: Q_{j} \rightarrow \mathbb{C}, j=1, \ldots, 4$, denote these solutions with $Q_{j}=e^{(2 j-1) \pi i / 4} Q$, where $Q$ is the image of $\Omega(M, r, \gamma)$ (with certain $M, r, \gamma>0$ ) by $x \mapsto x^{1 / 2}$ introduced above Theorem 2.2. The domain $Q$ is shown on Figure 2.2 (it has the name $Q_{1}$ there). Since $\arg \varepsilon=\frac{\pi}{2}$, the domains $Q_{1}, \ldots, Q_{4}$ are now rotated by an angle of $\frac{\pi}{4}$ compared to the case $\arg \varepsilon=0$ of Proposition 2.3.

Analogously to the beginning of this section, $p_{+}^{\infty}=v_{-}^{\infty} \circ y_{+}^{\infty}-$ id can be defined in some sector $\mathcal{I}_{+}=\left\{x \in \mathbb{C} ;|\arg (-x-L)|<\frac{\pi}{2}-\delta\right\}$, where $L, \delta>0$. It can be shown by analytic continuation that $L>0$ can be chosen small. Then $p_{+}^{\infty}$ can be analytically continued by periodicity from $\mathcal{I}_{+}$to the half plane $H_{L}=\{x \in \mathbb{C} ; \operatorname{Re} x<-L\}$ with small positive $L$. If $L$ is small enough, then $H_{L}$ has a nonempty intersection with the above rhombus $K$. On $K \cap \mathcal{I}_{+}$, we can write $v_{-}^{\infty} \circ y_{+}^{\infty}=\left(v_{-}^{\infty} \circ y_{\alpha}\right) \circ\left(v_{\alpha} \circ y_{+}^{\infty}\right)=$ $\left(\mathbf{i d}+p_{\alpha-}\right) \circ\left(\mathbf{i d}+p_{\alpha+}\right)$ with some $\varepsilon$-periodic functions $p_{\alpha \pm}$.

Now these functions $p_{\alpha \pm}$ can be studied as before by continuing $v_{\alpha}, v_{ \pm}^{\infty}$ analytically. If $\delta$ is small enough, then $M_{+}$has a nonempty intersection with $Q_{2}$, whereas $M_{-}$has nonempty intersection with $Q_{3}$. We can continue $v_{\alpha}$ analytically from $M_{+}$to all of $Q_{2}$, if $r$ is small enough. In order to still have a well defined function, we restrict this continuation to the intersection $Q_{2}^{+}=\left\{z \in Q_{2} ; \operatorname{Im} z>0\right\}$ of $Q_{2}$ with the upper half plane. Similarly, we analytically continue $v_{\alpha}$ from $M_{-}$to all of $Q_{3}^{-}$. As before $v_{+}^{\infty}$ can be continued analytically to $Q_{1}$ and $v_{-}^{\infty}$ can be continued analytically to $Q_{4}$.

There exist shifts $s_{\alpha \pm}(\varepsilon)$ such that $v_{\alpha}(z)-v_{2}^{*}(z)-s_{\alpha+}(\varepsilon)$, resp. $v_{\alpha}(z)-v_{3}^{*}(z)-$ $s_{\alpha-}(\varepsilon)$ are exponentially small on $Q_{2}^{+}$, resp. $Q_{3}^{-}$. We have shown above that $v_{+}^{\infty}(z)-$ $v_{1}^{*}(z)-s(\varepsilon)$ is exponentially small for $s(\varepsilon)=\frac{1}{2}(q(\varepsilon)-1) \log (2 \varepsilon)=-\frac{\varepsilon}{4} \log (2 \varepsilon)(1+$ $o(1))$. Analogously $v_{-}^{\infty}(z)-v_{4}^{*}(z)-\widetilde{s}(\varepsilon)$ turns out to be exponentially small for $\widetilde{s}(\varepsilon)=$ $\frac{1}{2}(q(\varepsilon)-1) \log (2 \varepsilon)-2 \pi i q(\varepsilon)$. This yields $p_{\alpha-}(x, \varepsilon)=p_{3}\left(x-s_{\alpha-}(\varepsilon), \varepsilon\right)+\widetilde{s}(\varepsilon)-$ $s_{\alpha-}(\varepsilon)+\mathcal{O}\left(e^{-c /|\varepsilon|}\right)$ and $p_{\alpha+}(x, \varepsilon)=p_{1}(x-s(\varepsilon), \varepsilon)-s(\varepsilon)+s_{\alpha+}(\varepsilon)+\mathcal{O}\left(e^{-c /|\varepsilon|}\right)$ where $p_{1}, p_{3}$ are the Écalle-Voronin invariants of (7.1) and $c$ is some positive constant. Because of id $+p_{+}^{\infty}=\left(\mathbf{i d}+p_{\alpha-}\right) \circ\left(\mathbf{i d}+p_{\alpha+}\right)$, we have altogether ${ }^{4}$

$$
p_{+}^{\infty}(x)=p_{1}(x-s)+p_{3}\left(x-s+p_{1}(x-s)+s_{\alpha+}-s_{\alpha-}\right)+s_{\alpha+}-s_{\alpha-}+\widetilde{s}-s .
$$

As we know that $p_{j}(x)$ are exponentially close to the sums of the positive powers of $e^{2 \pi i x / \varepsilon}$, i.e. to $\sum_{n \geq 0} c_{j n} e^{2 \pi i n x / \varepsilon}, j=1,3$, we can express the Fourier coefficients of $p_{+}^{\infty}$ by those of $p_{1}, p_{3}$ except for exponentially small terms. Especially we find

$$
c_{1+}^{\infty}(\varepsilon)=e^{-2 \pi i s(\varepsilon) / \varepsilon}\left(c_{1,1}(\varepsilon)+c_{3,1}(\varepsilon) e^{2 \pi i\left(c_{1,0}+s_{\alpha+-} s_{\alpha-}\right)(\varepsilon) / \varepsilon}\right)+\mathcal{O}\left(e^{-c /|\varepsilon|}\right) .
$$

4. We omit the dependence upon $\varepsilon$ here. 
The facts that $v_{1}^{*}$ and $v_{2}^{*}$ have the same outer expansion (2.14) and that they are Gevrey, imply that $c_{1,0}(\varepsilon)$ is exponentially small and it remains to determine $s_{\alpha+}-s_{\alpha-}$. This is done again using a Gevrey expansion of $v_{\alpha}$ and the outer expansions of $v_{2}^{*}, v_{3}^{*}$. Because of the different determinations of $\log (1+z)$ on $M_{ \pm}$, we find that $\left(s_{\alpha+}-s_{\alpha-}\right)(\varepsilon)=2 \pi i q(\varepsilon)$ and thus that altogether

$$
c_{1+}^{\infty}(\varepsilon)=e^{-\pi i(q(\varepsilon)-1) \log (2 \varepsilon) / \varepsilon}\left(c_{1,1}(\varepsilon)+c_{3,1}(\varepsilon) e^{-4 \pi^{2} q(\varepsilon) / \varepsilon}\right)+\mathcal{O}\left(e^{-c /|\varepsilon|}\right),
$$

where $c_{j, 1}, j=1,3$ are the Écalle-Voronin invariants of (7.1) at the origin which, according to Proposition 2.5, have Gevrey- $\frac{1}{2}$ asymptotic expansions

$$
c_{j, 1}(\varepsilon) \sim_{\frac{1}{2}} \sum_{n \geq 2} a_{j 1 n} \eta^{n}, j=1,3,
$$

where $a_{112}=C_{1}^{+}, a_{312}=e^{-\pi^{2}} C_{1}^{+}$and $C_{1}^{+}$is the first Écalle-Voronin invariant for the inner reduced equation (2.6), $\mathrm{cf}(2.10)$. It can be shown numerically that it does not vanish.

Since $(q(\varepsilon)-1) / \varepsilon$ is bounded, we see that $c_{1+}^{\infty}$ vanishes exponentially close to values of $\varepsilon$ where the sum of the two terms in the parenthesis vanishes. Our asymptotic expression implies that there is a sequence of such $\left(\varepsilon_{k}\right)_{k \in \mathbb{N}}$ and that (except for some integer shift) they satisfy

$$
\varepsilon_{k}^{-1} \sim_{1} \frac{1}{2 \pi i}\left(k+\frac{1}{2}\right)+\frac{1}{4}+\sum_{l \geq 1} \beta_{l} k^{-l / 2}
$$

with certain coefficients $\beta_{l}$.

\section{References}

[1] X. Buff, J. ÉCAlle, A. Epstein, "Limits of degenerate parabolic quadratic rational maps", Geom. Funct. Anal. num. 23, 2013, 42-95.

[2] J. ÉCALLE, "Théorie itérative: introduction à la théorie des invariants holomorphes", J. Math. Pures Appl. num. 54, 1975, 183-258.

[3] A. Fruchard, R. SCHÄFKE, "Analytic solutions of difference equations with small step size", J. Difference Eq. Appl. num. 7, 2001, 651-684.

[4] A. Fruchard, R. SChÄFKe, "Composite Asymptotic Expansions", Lect. Notes Math. num. 2066, Springer, 2013.

[5] J. Martinet, J.-P. Ramis, "Problèmes de modules pour des équations différentielles non linéaires du premier ordre”, Inst. Hautes Études Sci. Publ. Math. num. 55, 1982, 63-164.

[6] W. Rudin, Real and Complex Analysis, Third Edition, Mc Graw-Hill, 1987.

[7] S. M. Voronin, "Analytic classification of germs of conformal mappings $(\mathrm{C}, 0) \rightarrow(\mathrm{C}, 0)$ ", Funktsional. Anal. i Prilozhen num. 15, 1981, 1-17 (Russian; English translation: Functional Anal. Appl. num. 15, 1981, 1-13). 